\title{
RICCI FLOW ON THREE-DIMENSIONAL MANIFOLDS WITH SYMMETRY
}

\author{
JOHN LOTT AND NATASA SESUM
}

\begin{abstract}
We describe the Ricci flow on two classes of compact three-dimensional manifolds :

1. Warped products with a circle fiber over a two-dimensional base.

2. Manifolds with a free local isometric $T^{2}$-action.
\end{abstract}

\section{INTRODUCTION}

In understanding three-dimensional Ricci flow solutions, a special role is played by three-manifolds with symmetry. It is plausible that one can get more precise results about such Ricci flows than in the general case. Consequently, such Ricci flows with symmetry have been studied for quite a while. We begin by describing some of the earlier results.

Locally homogeneous three-dimensional Ricci flow solutions were examined by Isenberg-Jackson [IJ92] and Knopf-McLeod [KM01]. The flow equations reduced to a system of three coupled ODEs. The solutions are now fairly well understood; see, for example, LLot07, Section 3].

Certain three-dimensional Ricci flow solutions with a two-dimensional isometry group were analyzed by Carfora-Isenberg-Jackson [CIJ90] and Hamilton [Ham95, Section 11]. They considered Riemannian threemanifolds that admit a free isometric $T^{2}$-action. The base is a circle and the total space is (necessarily) diffeomorphic to a 3-torus. Under some additional assumptions $(\mathcal{F}$-metric [CIJ90] or square torus metric [Ham95, Section 11]) it was shown that the Ricci flow exists for all time and converges to a flat metric.

Hamilton and Isenberg considered a twisted version of such torus bundles [HI93. That is, there was a local (with respect to the base) free isometric $T^{2}$-action. The $T^{2}$-bundle over the circle was globally

Date: October 4, 2011.

2010 Mathematics Subject Classification. 53C44,57M50.

The research of the first author was partially supported by NSF grant DMS0900968. The research of the second author was partially supported by NSF grant DMS-1110145. 
twisted by a hyperbolic element of $\operatorname{SL}(2, \mathbb{Z})$; see Subsection 3.1 for a more precise description. Under the additional assumption of a "solvGowdy" metric, it was shown that the Ricci flow approaches that of a locally homogeneous Sol-metric; see also [Kno00].

Passing to one-dimensional isometry groups, a natural class of geometries comes from warped product metrics with circle fibers and a closed surface base $M$. One starts with a product metric on $N=$ $M \times S^{1}$ and then allows the circle length over $m \in M$ to become $m$ dependent. That is, we consider Riemannian metrics $h$ on $N$ of the form

$$
h=g+e^{2 u} d \theta^{2}
$$

where $g$ is a Riemannian metric on $M, u \in C^{\infty}(M)$ and $\theta$ is the standard coordinate on $S^{1}$. It is not hard to see that the Ricci flow preserves the warped product structure. The Ricci flow equation on $N$ becomes two coupled evolution equations on $M$ for $g(t)$ and $u(t)$. The evolution equation for $g(t)$ is like the Ricci flow equation on the surface $M$, but there is an extra term involving $u(t)$. Because of this extra term, the techniques used to analyze Ricci flow on surfaces [CK04, Chapter 5] break down.

When $M$ is a two-sphere, Xiaodong Cao showed that the product of $\mathbb{R}$ and a cigar soliton cannot arise as a finite-time dilation limit of a warped product Ricci flow on $S^{2} \times S^{1}$. Cao05. His argument used an isoperimetric inequality. (Cao's work preceded Perelman's proof that the product of $\mathbb{R}$ and a cigar soliton can never arise as a finite-time dilation limit for the Ricci flow on a compact three-manifold [Per02].)

In the present paper we use new techniques to give general results about the Ricci flow on three-manifolds with symmetries. One of our tools is the result of [Lot10] giving the long-time behavior of a threedimensional Ricci flow solution $(N, h(\cdot))$ satisfying $\max _{p \in N}\left|\mathrm{Rm}^{N}\right|(p, t)=$ $O\left(t^{-1}\right)$ and $\operatorname{diam}(N, h(t))=O(\sqrt{t})$. Thus one of our main goals is to show that these bounds are satisfied in the relevant cases. In particular, we show that $\max _{p \in N}\left|\mathrm{Rm}^{N}\right|(p, t)=O\left(t^{-1}\right)$ for all of the immortal Ricci flows under consideration. (A Ricci flow solution is immortal if it exists for $t \in[0, \infty)$.) It is an open question whether an immortal Ricci flow solution on a compact three-manifold always satisfies this curvature bound.

To describe the results of the paper, we start with warped products. In the special case of a product metric, the Ricci flow solution is the isometric product of $S^{1}$ with a Ricci flow solution on the base $M$. Such two-dimensional Ricci flows are well understood [CK04, Chapter 5]. If $h(0)$ is a warped product metric on $N=M \times S^{1}$ then a natural 
RICCI FLOW ON THREE-DIMENSIONAL MANIFOLDS WITH SYMMETRY 3

conjecture is that the Ricci flow asymptotically approaches a product flow.

Theorem 1.1. Let $h(\cdot)$ be a Ricci flow solution on a closed connected orientable three-dimensional manifold $N$. Suppose that $h(0)$ is a warped product metric as in (1.1) with a two-dimensional orientable base $M$.

(i) If $\chi(M)>0$ then there is a finite singularity time $T<\infty$. As $t \rightarrow T^{-}$, the lengths of the circle fibers remain uniformly bounded above and below. For any $p \in N$, the pointed smooth limit $\lim _{t \rightarrow T^{-}}\left(N, p, \frac{1}{T-t} h(t)\right)$ exists and is the isometric product of $\mathbb{R}$ with a sphere $S^{2}$ of constant curvature $\frac{1}{2}$.

(ii) If $\chi(M) \leq 0$ then the Ricci flow exists for all $t \in[0, \infty)$. Also, there is a constant $C<\infty$ so that for all $p \in N$ and $t \in[0, \infty)$, one has $\left|\mathrm{Rm}^{N}\right|(p, t) \leq \frac{C}{t}$.

(iii) If $\chi(M)=0$ then $\lim _{t \rightarrow \infty} h(t)$ exists and is a flat metric on $T^{3}$. The convergence is exponentially fast.

(iv) If $\chi(M)<0$, put $\widehat{g}(t)=\frac{g(t)}{t}$. For any $i_{0}>0$, define the $i_{0}$-thick part of $(M, \widehat{g}(t))$ by

$$
X_{i_{0}}(t)=\left\{m \in M: \operatorname{inj}_{\widehat{g}(t)}(m) \geq i_{0}\right\} .
$$

Then

$$
\lim _{t \rightarrow \infty} \max _{x \in X_{i_{0}}(t)}\left|R_{\widehat{g}(t)}(x)+1\right|=0
$$

and

$$
\lim _{t \rightarrow \infty} \max _{x \in X_{i_{0}}(t)}|\widehat{\nabla} u|_{\widehat{g}(t)}(x)=0 .
$$

For all sufficiently small $i_{0}$, if $t$ is sufficiently large then $X_{i_{0}}(t)$ is nonempty.

Remark 1.2. The proof of Theorem 1.1.(i) is essentially contained in List's paper [Lis08] on a modified Ricci flow. The only thing missing from [Lis08] is the observation that his flow differs from the warped product flow by a Lie derivative.

Remark 1.3. The proof of the curvature bound in Theorem 1.1.(ii) is by contradiction, using a blowup argument, a sharp volume estimate and the Gauss-Bonnet theorem.

Remark 1.4. The proof of Theorem 1.1.(iii) is somewhat indirect. We first show that $\operatorname{Vol}(M, g(t))=O(\sqrt{t})$ and that the length of the shortest noncontractible curve on $M$ is nondecreasing in $t$. A geometric 
argument then shows that $\operatorname{diam}(M, g(t))=O(\sqrt{t})$. From [Lot10, we deduce that

$$
\left(\max _{p \in N}\left|\mathrm{Rm}^{N}\right|(p, t)\right) \cdot \operatorname{diam}^{2}(N, h(t))=o(t) .
$$

Rescaling at a given time $t$ to diameter one, if $t$ is sufficiently large then we can assume that $\max _{p \in N}\left|\mathrm{Rm}^{N}\right|(p, t)$ is arbitrarily small. After passing to a finite cover $\widehat{N}$, we can assume that there is a universal lower bound on the injectivity radius of the pullback metric $\widehat{h}(t)$. By the linear stability of flat metrics GIK02, $\lim _{t \rightarrow \infty} \widehat{h}(t)$ exists and is a flat metric. Hence $\lim _{t \rightarrow \infty} h(t)$ exists and is a flat metric.

Remark 1.5. Theorem 1.1.(iv) says that in the case $\chi(M)<0$ and as $t \rightarrow \infty$, over a large part of $M$ the flow $(N, h(t))$ approaches a product flow of $S^{1}$ times a finite-volume surface of constant sectional curvature $-\frac{1}{2 t}$. Our result here is possibly nonoptimal; see Remark 2.20 .

Our other main result is about Ricci flow solutions with a local $U(1) \times$ $U(1)$ symmetry.

Theorem 1.6. Let $N$ be an orientable three-manifold that fibers over $S^{1}$ with $T^{2}$-fibers. Choosing an orientation for $S^{1}$, let $H \in \mathrm{SL}(2, \mathbb{Z})=$ $\pi_{0}\left(\operatorname{Diff}^{+}\left(T^{2}\right)\right)$ be the holonomy of the torus bundle. We can consider $N$ to be the total space of a twisted principal $U(1) \times U(1)$ bundle, where the twisting is determined by $H$.

Let $h(\cdot)$ be a Ricci flow solution on $N$. Suppose that $h(0)$ is invariant under the local $U(1) \times U(1)$ actions. Then the Ricci flow exists for all $t \in[0, \infty)$. There is a constant $C<\infty$ so that for all $p \in N$ and $t \in[0, \infty)$, one has $\left|\mathrm{Rm}^{N}\right|(p, t) \leq \frac{C}{t}$.

(i) If $H$ is elliptic, i.e. has finite order, then $\lim _{t \rightarrow \infty} h(t)$ exists and is a flat metric on $N$. The convergence is exponentially fast.

(ii) Suppose that $H$ is hyperbolic, i.e. has two distinct real eigenvalues. We write $h(t)$ in the form

$$
h(t)=g_{y y}(y, t) d y^{2}+(d x)^{T} G(y, t) d x,
$$

where $\left\{x^{1}, x^{2}\right\}$ are local coordinates on $T^{2}$ and $y \in[0,1)$ is a local coordinate on $S^{1}$. Then up to an overall change of parametrizations for $S^{1}$ and $T^{2}$, we have

$$
\begin{aligned}
\lim _{t \rightarrow \infty} \frac{g_{y y}(y, t)}{t} & =\frac{1}{2} \operatorname{Tr}\left(X^{2}\right), \\
\lim _{t \rightarrow \infty} G(y, t) & =e^{y X},
\end{aligned}
$$


RICCI FLOW ON THREE-DIMENSIONAL MANIFOLDS WITH SYMMETRY 5

where $X$ is the real symmetric matrix such that $e^{X}=H^{T} H$. The convergence in (1.7) is power-decay fast in $t$.

Remark 1.7. Theorem 1.6, (ii) says that $(N, h(\cdot))$ approaches a locally homogeneous Ricci flow solution of Sol-type. The proof of Theorem 1.6. (ii) uses the monotonicity of the modified $W_{+}$-functional from Lot10, Section 4.2.3], along with the local stability result of [Kno09, Theorem $3]$.

Remark 1.8. Theorem 1.6. (i) includes the cases considered in [CIJ90] and [Ham95, Section 11]. Theorem 1.6.(ii) includes the case considered in [HI93].

The structure of the paper is as follows. In Section 2 we prove Theorem 1.1. In Section 3 we prove Theorem 1.6. More detailed descriptions are at the beginnings of the sections.

We are grateful to Jim Isenberg for introducing us to these problems and for sharing his knowledge with us. We learned the estimates (2.19), (2.24) and (2.26) from Jim.

In what follows we will use the Einstein summation convention freely.

\section{WARPED PRODUCTS}

In this section we prove Theorem 1.1. In Subsection 2.1 we write the warped product Ricci flow equations and the corresponding evolution equations for geometric quantities. In Subsection 2.2 we give a priori bounds using the maximum principle and integral estimates. In Subsection 2.3 we deal with the case $\chi(M)>0$. In Subsection 2.4 we prove that the Ricci flow exists for $t \in[0, \infty)$ when $\chi(M) \leq 0$. In Subsection 2.5 we deal with the case $\chi(M)=0$. In Subsection 2.6 we deal with the case $\chi(M)<0$.

2.1. Warped product Ricci flow. Let $N=M \times S^{1}$ be endowed with a warped product metric

$$
h=g+e^{2 u} d \theta^{2} .
$$

Here $g$ is a Riemannian metric on $M, u$ is a smooth function on $M$ and $\theta \in[0,2 \pi)$. We use $i, j, k, l$ for the indices on $M$. Nonzero components of the curvature tensor of $(N, h)$ are

$$
\begin{aligned}
& R_{i j k l}^{N}=R_{i j k l}^{M}, \\
& R_{\theta i \theta j}^{N}=-e^{2 u}\left[\nabla_{i} \nabla_{j} u+\left(\nabla_{i} u\right)\left(\nabla_{j} u\right)\right] .
\end{aligned}
$$


Its square norm is

$$
\begin{aligned}
\left|\mathrm{Rm}_{h}^{N}\right|^{2}= & \left|\mathrm{Rm}_{g}^{M}\right|^{2}+ \\
& 2 g^{i i^{\prime}} g^{j j^{\prime}}\left[\nabla_{i} \nabla_{j} u+\left(\nabla_{i} u\right)\left(\nabla_{j} u\right)\right]\left[\nabla_{i^{\prime}} \nabla_{j^{\prime}} u+\left(\nabla_{i^{\prime}} u\right)\left(\nabla_{j^{\prime}} u\right)\right] .
\end{aligned}
$$

We will often think of $\left|\operatorname{Rm}_{h}^{N}\right|(\cdot, t)$ as a function on $M$ rather than $N$, since it pulls back from $M$, and write $\left|\operatorname{Rm}_{h}^{N}\right|(m, t)$. Note that a sectional curvature bound on $(N, h)$ implies the same sectional curvature bound on $(M, g)$.

The nonzero components of the Ricci tensor are

$$
\begin{aligned}
& \operatorname{Ric}_{i j}^{N}=\operatorname{Ric}_{i j}^{M}-\nabla_{i} \nabla_{j} u-\left(\nabla_{i} u\right)\left(\nabla_{j} u\right), \\
& \operatorname{Ric}_{\theta \theta}^{N}=-e^{2 u}\left(\triangle u+|\nabla u|^{2}\right) .
\end{aligned}
$$

The scalar curvature is

$$
R^{N}=R^{M}-2 \triangle u-2|\nabla u|^{2} .
$$

The Ricci flow equation

$$
\frac{d h}{d t}=-2 \operatorname{Ric}_{h(t)}
$$

on $N$ preserves the warped product structure. In terms of $g$ and $u$, it becomes

$$
\begin{aligned}
\frac{\partial g_{i j}}{\partial t} & =-2 R_{i j}+2 \nabla_{i} \nabla_{j} u+2\left(\nabla_{i} u\right)\left(\nabla_{j} u\right), \\
\frac{\partial u}{\partial t} & =\Delta u+|\nabla u|^{2},
\end{aligned}
$$

where $\Delta=\Delta_{g(t)}$. Adding the Lie derivative with respect to $-\nabla u$ to the right-hand side of (2.7) gives the modified equations

$$
\begin{aligned}
\frac{\partial g_{i j}}{\partial t} & =-2 R_{i j}+2\left(\nabla_{i} u\right)\left(\nabla_{j} u\right), \\
\frac{\partial u}{\partial t} & =\Delta u .
\end{aligned}
$$

Hereafter we will mainly work with the system (2.8), since geometric statements about (2.8) will imply the corresponding statements about the Ricci flow (2.7).

Given $s>0$ and a solution $(g(\cdot), u(\cdot))$ of (2.8), we obtain another solution $\left(g_{s}(\cdot), u_{s}(\cdot)\right)$ of (2.8) by putting

$$
\begin{aligned}
& g_{s}(t)=\frac{1}{s} g(s t), \\
& u_{s}(t)=u(s t) .
\end{aligned}
$$


Note that the rescaling in (2.9) differs from the three-dimensional rescaling of the Ricci flow $(N, h(\cdot))$, which would give

$$
\begin{aligned}
G_{s}(t) & =\frac{1}{s} g(s t), \\
U_{s}(t) & =u(s t)-\frac{1}{2} \ln (s) .
\end{aligned}
$$

The rescaling in (2.9) can be interpreted in the following way. There is a $\mathbb{Z}$-cover $\widehat{N}=M \times \mathbb{R}$ of $N$ with a pullback Ricci flow $\widehat{h}(\cdot)$. On $\widehat{N}$, the rescaling in (2.9) amounts to looking at the Ricci flow solution $\frac{1}{s} \phi_{s}^{*} \widehat{h}(s t)$, where $\phi_{s}(m, r)=(m, \sqrt{s} r)$. We refer to [Lot07, Section 4] for further discussion of this point.

Put

$$
h_{s}=g_{s}+e^{2 u_{s}} d \theta^{2} .
$$

One sees from (2.3) and (2.9) that

$$
\left|\mathrm{Rm}_{h_{s}}\right|^{2}=s^{2}\left|\mathrm{Rm}_{h}\right|^{2} .
$$

This can also be seen from the discussion of the previous paragraph on $\widehat{N}$ along with the fact that under the three-dimensional rescaling of (2.10), the norm square of the curvature gets multiplied by $s^{2}$.

Following [Lis08], we introduce the tensor

$$
S_{i j}=R_{i j}-\left(\nabla_{i} u\right)\left(\nabla_{j} u\right)
$$

and its trace

$$
S=R-|\nabla u|^{2}
$$

Putting $\alpha_{n}=1$ in [Lis08, Lemma 3.2] gives

$$
\frac{\partial|\nabla u|^{2}}{\partial t}=\triangle|\nabla u|^{2}-2|\operatorname{Hess}(u)|^{2}-2|\nabla u|^{4}
$$

and

$$
\frac{\partial S}{\partial t}=\triangle S+2\left|S_{i j}\right|^{2}+2|\triangle u|^{2}
$$

Hereafter we specialize to the case when $M$ is closed, connected and orientable, with $\operatorname{dim}(M)=2$. Then (2.8) simplifies to

$$
\begin{aligned}
\frac{\partial g_{i j}}{\partial t} & =-R g_{i j}+2\left(\nabla_{i} u\right)\left(\nabla_{j} u\right), \\
\frac{\partial u}{\partial t} & =\Delta u .
\end{aligned}
$$

Note that if $u$ is nonconstant then the conformal class of $g(t)$ is $t$ dependent. 


\subsection{A priori bounds.}

2.2.1. Bounds from the maximum principle. We take the Ricci flow to start at time zero. Applying the maximum principle to the second equation in (2.8) shows that there exist constants $C_{1}, C_{2}>0$ so that for all $m \in M$ and all $t$ for which the flow exists,

$$
C_{1} \leq u(m, t) \leq C_{2} .
$$

Next, applying the maximum principle to (2.15) implies (c.f. Lis08, Lemma 5.6]) that

$$
|\nabla u|^{2}(\cdot, t) \leq \frac{c}{2 c t+1}
$$

where $c=\max _{p \in M}|\nabla u|^{2}(p, 0)$. Similarly, applying the maximum principle to (2.16) implies that

$$
S(\cdot, t) \geq-\frac{1}{t}
$$

In particular,

$$
R(\cdot, t) \geq-\frac{1}{t}
$$

2.2.2. Volume estimates. Let $V(t)$ denote the volume of $\left(M^{2}, g(t)\right)$. As

$$
\frac{d}{d t} d V_{g(t)}=\frac{1}{2} g^{i j} \frac{\partial g_{i j}}{\partial t} d V_{g(t)}=\left(-R+|\nabla u|^{2}\right) d V_{g(t)},
$$

we have

$$
\frac{d V}{d t}=-4 \pi \cdot \chi(M)+\int_{M}|\nabla u|^{2} d V_{g(t)},
$$

where $\chi(M)$ is the Euler characteristic of $M$. Thus $\frac{d V}{d t} \geq-4 \pi \cdot \chi(M)$ and so

$$
V(t) \geq-4 \pi \cdot \chi(M) \cdot t+V(0) .
$$

Using (2.19) in addition, we can control $V(t)$ from above. Namely, from (2.19),

$$
\frac{d V}{d t} \leq-4 \pi \cdot \chi(M)+\frac{c}{2 c t+1} \cdot V
$$

where $c$ is as in (2.19). Then by ODE comparison,

$$
V(t) \leq-\frac{4 \pi}{c} \cdot \chi(M) \cdot(2 c t+1)+\sqrt{2 c t+1} \cdot\left(\frac{4 \pi \cdot \chi(M)}{c}+V(0)\right)
$$

If $\chi(M)=0$ then estimates (2.24) and (2.26) imply that

$$
V(0) \leq V(t) \leq C(1+\sqrt{t})
$$


for an appropriate constant $C<\infty$.

When $\chi(M)<0$, the linear term on the right-hand side of $(2.26)$ is $-8 \pi \chi(M) t$. We would like to improve this to $-4 \pi \chi(M) t$, to bring it in line with (2.24). Put $E(t)=\int_{M}|\nabla u|^{2} d V_{g(t)}$.

Lemma 2.1. We have

$$
\frac{d E}{d t} \leq-\frac{E^{2}(t)}{V(t)}
$$

Proof. First, as in (2.22), the volume density changes by

$$
\frac{d}{d t}\left(d V_{g(t)}\right)=-S d V_{g(t)}
$$

Note that

$$
\int_{M} S d V_{g(t)}=\int_{M}\left(R-|\nabla u|^{2}\right) d V_{g(t)}=4 \pi \chi(M)-E(t) .
$$

Then

$$
\begin{aligned}
-\frac{d E}{d t} & =\frac{d}{d t} \int_{M} S d V_{g(t)}=\int_{M}\left(\Delta S+2\left|S_{i j}\right|^{2}+2(\Delta u)^{2}-S^{2}\right) d V_{g(t)} \\
& =2 \int_{M}\left|S_{i j}-\frac{1}{2} S g_{i j}\right|^{2} d V_{g(t)}+2 \int_{M}(\Delta u)^{2} d V_{g(t)} \\
& =\int_{M}\left(\left.\left.2\left|-\nabla_{i} u \nabla_{j} u+\frac{1}{2}\right| \nabla u\right|^{2} g_{i j}\right|^{2}+2(\Delta u)^{2}\right) d V_{g(t)} \\
& =\int_{M}\left(|\nabla u|^{4}+2(\Delta u)^{2}\right) d V_{g(t)} \\
& \geq \int_{M}|\nabla u|^{4} d V_{g(t)} \\
& \geq \frac{1}{V(t)}\left(\int_{M}|\nabla u|^{2} d V_{g(t)}\right)^{2},
\end{aligned}
$$

which proves the lemma.

We now show that $E(t)$ decays logarithmically in time when $\chi(M)<$ 0 .

Corollary 2.2. If $\chi(M)<0$ then there exist constants $A, B>0$ so that

$$
\int_{M}|\nabla u|^{2} d V_{g(t)} \leq \frac{A}{1+B \ln (t+1)}
$$

at all $t$ for which the flow exists. 
Proof. By Lemma 2.1 and the volume estimate (2.26) we have

$$
\frac{d E}{d t} \leq-\frac{E^{2}(t)}{V(t)} \leq-\frac{E^{2}(t)}{c_{1} t+c_{2}}
$$

for appropriate constants $c_{1}, c_{2}>0$. The corollary follows from ODE comparison.

Corollary 2.3. If $\chi(M)<0$ then there is a function $\alpha:[0, \infty) \rightarrow$ $[0, \infty)$, with $\lim _{t \rightarrow \infty} \alpha(t)=0$, such that

$$
\left|\frac{V(t)}{-4 \pi \chi(M) \cdot(t+1)}-1\right| \leq \alpha(t)
$$

at all $t$ for which the flow exists.

Proof. This follows from (2.23) and (2.32).

Lemma 2.4. The quantity $\frac{V(t)}{t}$ is nonincreasing along the flow (2.8).

Proof. From (2.20) and (2.29),

$$
\frac{d}{d t}\left(\frac{V(t)}{t}\right)=-\frac{1}{t} \int_{M}\left(S+\frac{1}{t}\right) d V_{g(t)} \leq 0 .
$$

This proves the lemma.

In the sequel we will distinguish between the cases $\chi(M)>0$, $\chi(M)=0$ and $\chi(M)<0$.

\subsection{Positive Euler characteristic.}

Proposition 2.5. If $\chi(M)>0$ then there is a finite singularity time $T<\infty$. For any $p \in N$, the pointed smooth limit $\lim _{t \rightarrow T^{-}}\left(N, p, \frac{1}{T-t} h(t)\right)$ exists and is the isometric product of $\mathbb{R}$ with a sphere $S^{2}$ of constant curvature $\frac{1}{2}$.

Proof. If a smooth flow existed for all $t \in[0, \infty)$ then equation (2.26) would imply that $V(t)<0$ for large $t$, which is impossible. Thus there is a singularity at some time $T<\infty$.

From [Lis08, Theorem 5.15], $\lim _{t \rightarrow T^{-}} \max _{m \in M}|R|(m, t)=\infty$, where $R$ is the scalar curvature of $M$. Let $\left\{t_{k}\right\}_{k=1}^{\infty}$ be a sequence of times so that for sufficiently large $k, t_{k}$ is the first time $t$ for which $\max _{m \in M}|R|(m, t)=$ $k$. Let $m_{k} \in M$ be such that $|R|\left(m_{k}, t_{k}\right)=k$. From [Lis08, Theorem 7.9], a subsequence of the rescaled pointed solutions

$$
\left(M, m_{k}, g_{k}(t), u_{k}(t)\right)=\left(M, m_{k}, k g\left(t_{k}+t / k\right), u\left(t_{k}+t / k\right)\right)
$$

converges smoothly to a solution $\left(M_{\infty}, m_{\infty}, g_{\infty}(\cdot), u_{\infty}(\cdot)\right)$ defined for $t \in(-\infty, 0]$, where $g_{\infty}(\cdot)$ is a $\kappa$-solution on $M_{\infty}$ in the sense of [Per02, Section 11.1] and $u_{\infty}(\cdot)$ is constant both spatially and temporally. The 
proof of this statement uses a modified $W$-functional which was introduced in [Lis08] and [Lot10], and which becomes

$$
W(g, u, f, \tau)=\int_{M}\left[\tau\left(|\nabla f|^{2}+R-|\nabla u|^{2}\right)+f-2\right](4 \pi \tau)^{-1} e^{-f} d V_{g(t)} .
$$

in our three-dimensional warped product case.

The only $\kappa$-solution on an orientable surface is the round shrinking 2sphere [KL08, Corollary 40.1]. In particular, $\lim _{k \rightarrow \infty}\left(M, k g\left(t_{k}\right), u\left(t_{k}\right)\right)=$ $\left(S^{2}, g_{S^{2}}, u_{\infty}\right)$, where $g_{S^{2}}$ has constant scalar curvature one. (Because $S^{2}$ is compact, we no longer have to refer to basepoints. At this point our convergence is still modulo diffeomorphisms.) Standard arguments show that the system (2.7) is stable around the solution given by the round shrinking $S^{2}$ and constant $u$, with exponential convergence for the normalized flow. It follows that $\lim _{t \rightarrow T^{-}}\left(M, \frac{1}{T-t} g(t), u(t)\right)=$ $\left(S^{2}, g_{S^{2}}, u_{\infty}\right)$, where the convergence is now taken without diffeomorphisms.

In terms of the three-dimensional geometry, from (2.18) the fiber lengths are uniformly bounded above and below by positive constants, up to time $T$. The three-dimensional pointed $\operatorname{limit}_{t \rightarrow T^{-}}\left(N, \frac{1}{T-t} h(t), p\right)$ is the isometric product of $\mathbb{R}$ with $\left(S^{2}, g_{S^{2}}\right)$. This proves the proposition.

Remark 2.6. We could also prove Proposition 2.5 by looking at the three-dimensional singularity models with a nowhere-vanishing Killing vector field. Such a proof would be less elementary, since it would use the results of [Per02, Section 11].

Remark 2.7. The method of proof of Proposition 2.5 works for finitetime singularities of warped products $M \times S^{1}$ if $M$ is a compact manifold of arbitrary dimension $n-1$. Blowing up at points of maximal curvature, one obtains an $(n-1)$-dimensional ancient solution which is $\kappa$-noncollapsed at all scales.

\subsection{Nonsingularity when $\chi(M) \leq 0$.}

Proposition 2.8. If $\chi\left(M^{2}\right) \leq 0$ then the Ricci flow exists for $t \in$ $[0, \infty)$.

Proof. If not then there is a singularity at some time $T<\infty$. The same argument as in Subsection 2.3 gives $\lim _{k \rightarrow \infty}\left(M, k g\left(t_{k}\right), u\left(t_{k}\right)\right)=$ $\left(S^{2}, g_{S^{2}}, u_{\infty}\right)$. In particular, $M$ is diffeomorphic to $S^{2}$, which contradicts our assumption.

\subsection{Vanishing Euler characteristic.}


Proposition 2.9. If $\chi(M)=0$ then $\lim _{t \rightarrow \infty} h(t)$ exists and is a flat metric on $T^{3}$. The convergence is exponentially fast.

Proof. We first show in the following two propositions that if $\chi(M)=0$ then $\max _{p \in N}\left|\mathrm{Rm}^{N}\right|(p, t)=O\left(t^{-1}\right)$ and $\operatorname{diam}(N, h(t))=O(\sqrt{t})$. Here $\mathrm{Rm}^{N}$ denotes the three-dimensional sectional curvatures.

Proposition 2.10. If $\chi(M)=0$ then there is a $C<\infty$ so that for all $t \in[0, \infty)$, we have

$$
t \cdot \max _{p \in N}\left|\operatorname{Rm}^{N}\right|(p, t) \leq C .
$$

Proof. If not, $\lim \sup _{t \rightarrow \infty} t \cdot \max _{m \in M}\left|\mathrm{Rm}^{N}\right|(m, t)=\infty$. (Since the function $\left|\mathrm{Rm}^{N}\right|(\cdot, t)$ pulls back from $M$, we can think of it as a function on $M$.) We perform an analog of Hamilton's pointpicking algorithm for a type IIb Ricci flow solution; see [CLN06, Chapter 8.2.1.3]. Namely, take any sequence $\left\{T_{i}\right\}_{i=1}^{\infty}$ with $\lim _{i \rightarrow \infty} T_{i}=\infty$ and let $\left(m_{i}, t_{i}\right) \in M \times$ $\left[0, T_{i}\right]$ be such that

$$
t_{i}\left(T_{i}-t_{i}\right)\left|\mathrm{Rm}^{N}\right|\left(m_{i}, t_{i}\right)=\sup _{(m, t) \in M \times\left[0, T_{i}\right]} t\left(T_{i}-t\right)\left|\mathrm{Rm}^{N}\right|(m, t)
$$

Dilate the flow in space and time by $Q_{i}=\left|\mathrm{Rm}^{N}\right|\left(m_{i}, t_{i}\right)$ in the following way :

$$
g_{i}(t)=Q_{i} \cdot g\left(t_{i}+t Q_{i}^{-1}\right), \quad u_{i}(t)=u\left(t_{i}+t Q_{i}^{-1}\right) .
$$

Then $\left(g_{i}(\cdot), u_{i}(\cdot)\right)$ satisfies (2.8) on a time interval $\left(A_{i}, \Omega_{i}\right)$, with $\lim _{i} A_{i}=$ $-\infty$ and $\lim _{i} \Omega_{i}=\infty$. Put

$$
h_{i}(t)=g_{i}(t)+e^{2 u_{i}} d \theta^{2} .
$$

By construction, $\left|\operatorname{Rm}_{h_{i}}^{N}\right|\left(m_{i}, 0\right)=1$ and after redefining $A_{i}$ and $\Omega_{i}$, there is a sequence $\left\{\gamma_{i}\right\}_{i=1}^{\infty}$ with $\lim _{i \rightarrow \infty} \gamma_{i}=1$ such that

$$
\max _{(m, t) \in M \times\left[A_{i}, \Omega_{i}\right]}\left|\operatorname{Rm}_{h_{i}}^{N}\right|(m, t) \leq \gamma_{i} .
$$

The curvature bound on $N$ implies a curvature bound on $M$ which, along with the a priori bounds on $u_{i}$ and $\left|\nabla u_{i}\right|$, implies higher derivative bounds on $\mathrm{Rm}^{M}\left(g_{i}\right)$ and $u_{i}$ [Lis08, Theorem 5.12]. We would now like to take a convergent subsequence of the pointed flows $\{(M \times$ $\left.\left.\left[A_{i}, \Omega_{i}\right],\left(m_{i}, 0\right), g_{i}(\cdot), u_{i}(\cdot)\right)\right\}_{i=1}^{\infty}$ to obtain an eternal solution $\left(M_{\infty} \times\right.$ $\left.\mathbb{R},\left(m_{\infty}, 0\right), g_{\infty}(\cdot), u_{\infty}(\cdot)\right)$ of $(2.8)$, where $\mathbb{R}$ denotes a time interval. To do so, we need a uniform positive lower bound on the injectivity radius at $\left(m_{i}, 0\right)$. If the two-dimensional manifolds $\left(M, g_{i}(0)\right)$ were positively curved then such a bound would be automatic. Since we don't know that $\left(M, g_{i}(0)\right)$ is positively curved, we argue differently. 
Lemma 2.11. There is some $\epsilon>0$ such that for all $i$, the injectivity radius of $\left(M, g_{i}(0)\right)$ at $m_{i}$ is bounded below by $\epsilon$.

Proof. Suppose that the lemma is false. Then after passsing to a subsequence, we can assume that $\lim _{i \rightarrow \infty} \operatorname{inj}_{g_{i}(0)}\left(m_{i}\right)=0$. Passing to a further subsequence, we can assume that $\left\{\left(M \times\left[A_{i}, \Omega_{i}\right],\left(m_{i}, 0\right), g_{i}(\cdot), u_{i}(\cdot)\right)\right\}_{i=1}^{\infty}$ converges to a solution of (2.8) on an étale groupoid. For information about the use of étale groupoids in Ricci flow, we refer to [Lot07] and [Lot10]. The upshot is that after passing to a subsequence, we have smooth pointed convergence to an eternal solution $\left(M_{\infty} \times \mathbb{R},\left(m_{\infty}, 0\right), g_{\infty}(\cdot), u_{\infty}(\cdot)\right)$ of (2.8), where $M_{\infty}$ is a two-dimensional étale groupoid. Furthermore, this solution has uniformly bounded curvature by (2.42), and for each $t \in \mathbb{R},\left(M_{\infty}, g_{\infty}(t)\right)$ is a complete closed effective Riemannian groupoid.

By assumption, $\lim _{i \rightarrow \infty} t_{i} Q_{i}=\infty$. As

$$
\max _{m \in M}\left|\nabla u_{i}\right|_{g_{i}}^{2}(m, t)=Q_{i}^{-1} \max _{m \in M}|\nabla u|_{g}^{2}\left(m, t_{i}+t Q_{i}^{-1}\right)
$$

when combined with (2.19) we conclude that $\nabla u_{\infty}=0$ on any time slice. Then $u_{\infty}$ is constant spatially and temporally, and $g_{\infty}$ is just a Ricci flow on $M_{\infty}$. As it is an eternal solution, it has nonnegative curvature, with positive curvature at $\left(m_{\infty}, 0\right)$.

We know that $\left(M_{\infty}, m_{\infty}, g_{\infty}(t)\right)$ is a bounded curvature limit of $\left\{\left(M, m_{i}, g_{i}(t)\right)\right\}_{i=1}^{\infty}$. The Riemannian groupoid $\left(M_{\infty}, g_{\infty}(t)\right)$ has a locally constant sheaf of finite dimensional Lie algebras $\mathfrak{g}$ which act as germs of Killing vector fields on the unit space $M_{\infty}^{(0)}$. Because of the bounded curvature assumption, these local Killing vector fields do not have a point of common vanishing; see, for example, Ron07, Theorem 5.1].

As $M_{\infty}^{(0)}$ is two-dimensional, and we are in the collapsing situation, the only possibilities for $\mathfrak{g}$ are $\mathbb{R}^{2}$ and $\mathbb{R}$. If $\mathfrak{g}=\mathbb{R}^{2}$ then $\left(M_{\infty}, g_{\infty}(t)\right)$ is flat, which is a contradiction.

Suppose that $\mathfrak{g}=\mathbb{R}$. Locally, $g_{\infty}(t)$ can be written as $d x^{2}+f^{2}(x, t) d y^{2}$. Since its curvature is $K=-\frac{f^{\prime \prime}}{f}$, the function $f$ is concave. Fixing the value of $f$ at a single point in $M_{\infty}^{(0)}$, the function $f$ pulls back from a function on the orbit space $\mathcal{O}$ of $M_{\infty}$, which in our case is a onedimensional orbifold [Lot10, Proposition 5.2]. If $\mathcal{O}$ is a circle then we immediately get a contradiction, since the concave function $f$ must be constant, but this contradicts the fact that $\left(M_{\infty}, g_{\infty}\right)$ has nonzero curvature at $\left(m_{\infty}, 0\right)$. If $\mathcal{O}$ is an interval orbifold then we can pass to a double cover and argue as before. If $\mathcal{O}$ is $\mathbb{R}$ then the positive concave function $f$ must be constant, which again contradicts the fact 
that $\left(M_{\infty}, g_{\infty}\right)$ has nonzero curvature at $\left(m_{\infty}, 0\right)$. If $\mathcal{O}$ is $[0, \infty)$ then we can pass to a double cover and argue as before.

This proves the lemma.

We can now take a convergent subsequence of the pointed flows $\left\{\left(M \times\left[A_{i}, \Omega_{i}\right],\left(m_{i}, 0\right), g_{i}(\cdot), u_{i}(\cdot)\right)\right\}_{i=1}^{\infty}$ to obtain an eternal solution $\left(M_{\infty} \times\right.$ $\left.\mathbb{R},\left(m_{\infty}, 0\right), g_{\infty}(\cdot), u_{\infty}(\cdot)\right)$ of (2.8). From (2.19) and (2.43), $u_{\infty}$ is constant. Thus we have a nonflat eternal Ricci flow solution on the twodimensional manifold $M_{\infty}$, with uniformly bounded curvature (from (2.42) ) and complete time slices. In particular, $\int_{M_{\infty}} R_{g_{\infty}(0)} d V_{g_{\infty}(0)}>0$. Although we won't really need it, $\left(M_{\infty}, g_{\infty}(\cdot)\right)$ must be the cigar soliton, as follows from the fact that the spacetime supremum of $R$ is achieved at a point, along with a differential Harnack inequality CLN06, Theorem 9.4]. Hence

$$
\int_{M_{\infty}} R_{g_{\infty}(0)} d V_{g_{\infty}(0)}=4 \pi .
$$

For large $i$, there is a bounded domain $S_{i} \subset\left(M, g\left(t_{i}\right)\right)$ which, after rescaling, is almost isometric to a large piece of the time-zero slice of the cigar soliton. Then for large $i$,

$$
\int_{S_{i}} R_{g\left(t_{i}\right)} d V_{g\left(t_{i}\right)} \geq 3 \pi
$$

On the other hand,

$$
\int_{M-S_{i}} R_{g\left(t_{i}\right)} d V_{g_{i}(t)} \geq-\frac{1}{t_{i}} \operatorname{Vol}_{g\left(t_{i}\right)}\left(M-S_{i}\right) \geq-\frac{V\left(t_{i}\right)}{t_{i}} .
$$

Adding (2.45) and (2.46), and using (2.27), we see that for large $i$,

$$
\int_{M} R_{g\left(t_{i}\right)} d V_{g\left(t_{i}\right)} \geq 2 \pi
$$

This contradicts the assumption that $\chi(M)=0$ and proves Proposition 2.10

Proposition 2.12. $\operatorname{diam}(N, h(t))=O(\sqrt{t})$.

Proof. We will use the following general result about Riemannian submersions.

Lemma 2.13. If $\pi:(N, h) \rightarrow(M, g)$ is a Riemannian submersion, with $N$ compact and connected, then

$$
\operatorname{diam}(M, g) \leq \operatorname{diam}(N, h) \leq \operatorname{diam}(M, g)+2 \max _{m \in M} \operatorname{diam}\left(\pi^{-1}(m)\right),
$$

where $\operatorname{diam}\left(\pi^{-1}(m)\right)$ is the intrinsic diameter of $\pi^{-1}(m)$. 
Proof. Given $m_{1}, m_{2} \in M$, we have

$$
d_{M}\left(m_{1}, m_{2}\right)=d_{N}\left(\pi^{-1}\left(m_{1}\right), \pi^{-1}\left(m_{2}\right)\right) .
$$

It immediately follows that $\operatorname{diam}(M, g) \leq \operatorname{diam}(N, h)$.

Given $p_{1}, p_{2} \in N$, put $m_{1}=\pi\left(p_{1}\right)$ and $m_{2}=\pi\left(p_{2}\right)$. Then

$$
d_{N}\left(p_{1}, p_{2}\right) \leq \operatorname{diam}\left(\pi^{-1}\left(m_{1}\right)\right)+d_{N}\left(\pi^{-1}\left(m_{1}\right), \pi^{-1}\left(m_{2}\right)\right)+\operatorname{diam}\left(\pi^{-1}\left(m_{2}\right)\right) .
$$

The lemma follows.

From (2.18) and Lemma 2.13, $\operatorname{diam}(N, h(t)) \leq \operatorname{diam}(M, g(t))+2 e^{C_{2}}$. Thus it suffices to show that $\operatorname{diam}(M, g(t))=O(\sqrt{t})$.

Let $L(t)$ be the length (with respect to $g(t)$ ) of a shortest noncontractible closed geodesic $\gamma_{t}$ on $M$. We parametrize $\gamma_{t}$ by an arclength parameter $s$. We will first show the following claim, which is an analog of [Ham95, Theorem 12.1].

Claim 2.14. $L(t)$ is nondecreasing in $t$.

Proof. Given the curve $\gamma_{t}$ at time $t>0$, we obtain an upper bound on $L(t-\Delta t)$ by considering the length of the same curve $\gamma_{t}$ at the earlier time $t-\Delta t$. Then

$$
\begin{aligned}
\liminf _{\Delta t \rightarrow 0} \frac{L(t)-L(t-\Delta t)}{\Delta t} & \geq \frac{1}{2} \int_{0}^{L(t)} \frac{\partial g}{\partial t}\left(\gamma_{t}^{\prime}, \gamma_{t}^{\prime}\right) d s \\
& =\int_{0}^{L(t)}\left[-\frac{1}{2} R\left(\gamma_{t}(s)\right)+\left\langle\gamma_{t}^{\prime}, \nabla u\right\rangle^{2}\right] d s
\end{aligned}
$$

As $\gamma_{t}$ is stable, applying the second variation formula with respect to a parallel normal field along $\gamma_{t}$ gives

$$
-\int_{0}^{L(t)} R\left(\gamma_{t}(s)\right) d s \geq 0 .
$$

The claim follows.

Since $\chi(M)=0$, equations (2.27) and (2.38) imply that

$$
\lim _{t \rightarrow \infty}\left[\left(\max _{m \in M}|R|(m, t)\right) \cdot V(t)\right]=0 .
$$

The next claim is purely geometric and has nothing to do with flows.

Claim 2.15. There exist $\epsilon>0$ and $C<\infty$ so that for every metric $g$ on $M=T^{2}$ with $\left(\max _{m \in M}\left|R_{g}\right|(m)\right) \cdot \operatorname{Vol}(M, g) \leq \epsilon$, we have

$$
L(g) \cdot \operatorname{diam}(M, g) \leq C \cdot \operatorname{Vol}(M, g),
$$

where $L(g)$ is the length of the shortest noncontractible closed geodesic. 
Proof. Suppose first that the metric $g$ is flat. Let $\gamma$ be a shortest closed geodesic and let $\widehat{M}$ be the corresponding cyclic cover of $M$. Then $\widehat{M}=S^{1} \times \mathbb{R}$, where the circle has length $L(\gamma)=L(g)$. A generator of the covering group $\mathbb{Z}$ acts on $\widehat{M}$ by translation in the $\mathbb{R}$-direction, by some distance $L^{\prime}$, along with rotation around $S^{1}$ by some angle $\theta \in[-\pi, \pi]$. For any $\widehat{m} \in \widehat{M}$, we have

$$
d_{\widehat{M}}(\widehat{m}, g \widehat{m})=\sqrt{\left(L^{\prime}\right)^{2}+\left(\frac{\theta L}{2 \pi}\right)^{2}} .
$$

This is the length of a closed geodesic on $M$, so we must have

$$
\sqrt{\left(L^{\prime}\right)^{2}+\left(\frac{\theta L}{2 \pi}\right)^{2}} \geq L .
$$

This implies that $L^{\prime} \geq \frac{\sqrt{3}}{2} L$. Now $\operatorname{diam}(M, g) \leq \frac{1}{2}\left(L+L^{\prime}\right)$ and $\operatorname{Vol}(M, g)=L L^{\prime}$, so the claim holds in this case with $C=2$. Hence we can assume that $g$ is nonflat.

Suppose that the claim is false. Then there is a sequence $\epsilon_{i} \rightarrow 0$ so that for each $i$, there is a metric $g_{i}$ on $M$ with

$$
\left(\max _{m \in M}\left|R_{g_{i}}\right|(m)\right) \cdot \operatorname{Vol}\left(M, g_{i}\right) \leq \epsilon_{i}
$$

but

$$
L\left(g_{i}\right) \cdot \operatorname{diam}\left(M, g_{i}\right)>i \cdot \operatorname{Vol}\left(M, g_{i}\right) .
$$

Rescale $g_{i}$ so that $\max _{m \in M}\left|R_{g_{i}}\right|(m)=1$. Then $\operatorname{Vol}\left(M, g_{i}\right) \leq \epsilon_{i}$.

Let $\delta<<1$ be a new parameter. Suppose first that for an infinite number of $i$, the pointed Gromov-Hausdorff distance from $\left(M, m, g_{i}\right)$ to a point is greater than $\delta$ for all $m \in M$, i.e. $\operatorname{diam}\left(M, g_{i}\right)>\delta$. After relabelling the sequence $\left\{\left(M, g_{i}\right)\right\}_{i=1}^{\infty}$, we can assume that for all $i$ and all $m \in M$, the pointed Gromov-Hausdorff distance from $\left(M, m, g_{i}\right)$ to a point is greater than $\delta$. Since $\lim _{i \rightarrow \infty} \operatorname{Vol}\left(M, g_{i}\right)=0$, there is a sequence $\left\{c_{i}\right\}_{i=1}^{\infty}$ with $\lim _{i \rightarrow \infty} c_{i}=0$ so that for any $m \in M$, the pointed closed metric ball $\left(\overline{B\left(m, \frac{\delta}{10}\right)}, m, g_{i}\right)$ is $c_{i}$-close to $\left(\left[-\frac{\delta}{10}, \frac{\delta}{10}\right], 0\right)$ in the pointed Gromov-Hausdorff topology.

Fix $\delta^{\prime}<<1$. From [CFG92, for large $i$ there is a diffeomorphism $\phi_{i}$ from $\left(M, g_{i}\right)$ to $\left(S^{1} \times S^{1}, g_{i}^{\prime}\right)$, where

- $g_{i}^{\prime}$ is a warped product metric $d x^{2}+f_{i}^{2}(x) d y^{2}$,

- $\max _{x \in S^{1}} f_{i}(x) \rightarrow 0$ as $i \rightarrow \infty$, and

- $\phi_{i}$ is a $e^{\delta^{\prime}}$-biLipschitz map. 
More precisely, we are using the fact that the results of [CFG92] hold in a localized sense, i.e. without an upper diameter bound; see CT06, Section 2] for discussion. The paper CFG92 gives a biLipschitz approximation of $\left(M, g_{i}\right)$ by a Riemannian nilbundle with affine holonomy; see also [Fuk89]. In the present case such a Riemannian nilbundle is a Riemannian submersion $S^{1} \times S^{1} \rightarrow S^{1}$.

For the metric $g_{i}^{\prime}$, let $A_{i}$ be the length of the circle base. We know that $A_{i} \geq \frac{\delta}{10}$. To get an upper bound on $L\left(g_{i}\right)$, we locate a shortest circle fiber of the fiber bundle $S^{1} \times S^{1} \rightarrow S^{1}$ and take its preimage under $\phi_{i}$. This gives a noncontractible closed curve in $M$, so

$$
L\left(g_{i}\right) \leq e^{\delta^{\prime}} \min _{x \in S^{1}} f_{i}(x) .
$$

Next, using Lemma 2.13, the diameter of $\left(S^{1} \times S^{1}, g_{i}^{\prime}\right)$ is bounded above by $A_{i}+2 \max _{x \in S^{1}} f_{i}(x)$, so

$$
\operatorname{diam}\left(M, g_{i}\right) \leq e^{\delta^{\prime}}\left(A_{i}+2 \max _{x \in S^{1}} f_{i}(x)\right) .
$$

The volume of $\left(S^{1} \times S^{1}, g_{i}^{\prime}\right)$ is bounded below by $A_{i}$ times the length of the smallest circle fiber, so

$$
\operatorname{Vol}\left(M, g_{i}\right) \geq e^{-2 \delta^{\prime}} A_{i} \min _{x \in S^{1}} f_{i}(x)
$$

For large $i$, equations (2.59), (2.60) and (2.61) contradict (2.58).

Thus we can assume that for all but a finite number of $i$, there is some point $m_{i}$ so that the pointed Gromov-Hausdorff distance from $\left(M, m_{i}, g_{i}\right)$ to a point is at most $\delta$. We now rescale $\left(M, g_{i}\right)$ to a metric $\left(M, \widehat{g}_{i}\right)$ with diameter one. After this rescaling, $\max _{m \in M}\left|R_{\widehat{g}_{i}}\right|(m) \leq \delta^{2}$. Suppose that $\liminf \operatorname{in}_{i \rightarrow \infty} \operatorname{Vol}\left(M, \widehat{g}_{i}\right)=0$. After passing to a subsequence, we can assume that $\lim _{i \rightarrow \infty} \operatorname{Vol}\left(M, \widehat{g}_{i}\right)=0$. As in the argument of the preceding paragraphs, for large $i$ there is a biLipschitz approximation of $\left(M, \widehat{g}_{i}\right)$ by a warped product metric on a fiber bundle $S^{1} \times S^{1} \rightarrow S^{1}$, whose base has diameter close to one, and we obtain a contradiction to (2.58).

Thus there is some $v_{0}>0$ so that for all $i$, we have $\operatorname{Vol}\left(M, \widehat{g}_{i}\right) \geq v_{0}$. Cheeger compactness now gives a contradiction to (2.58). This proves the claim.

To conclude the proof of Proposition 2.12 we argue as follows. By (2.27), $V(t)=O(\sqrt{t})$. By (2.2) and Proposition 2.10, $\max _{m \in M}\left|R^{M}\right|(m, t)=$ $O\left(t^{-1}\right)$. Thus for large $t$, we can apply Claim 2.15 to conclude that

$$
\operatorname{diam}(M, g(t)) \leq C \frac{V(t)}{L(t)} \leq C \frac{V(t)}{L(0)},
$$


where Claim 2.14 is used in the last inequality. Proposition 2.12 follows.

By [Lot10, Theorem 1.2.1], Propositions 2.10 and 2.12 imply that $\max _{m \in M}\left|\operatorname{Rm}_{n}^{N}\right|(m, t)=o\left(t^{-1}\right)$. Thus

$$
\lim _{t \rightarrow \infty}\left[\left(\max _{m \in M}\left|\operatorname{Rm}^{N}\right|(m, t)\right) \cdot \operatorname{diam}^{2}(N, h(t))\right]=0 .
$$

For some given $t$, we rescale $(N, h(t))$ so that it has diameter one. We now wish to apply the local stability of flat metrics on $T^{3}$ under the Ricci flow. For this, we need the following lemma, which is purely geometric.

Claim 2.16. There are a compact subset $K$ of the moduli space of flat metrics on $T^{3}$ and a function $\epsilon^{\prime}:[0, \infty) \rightarrow[0, \infty)$, with $\lim _{\epsilon \rightarrow 0} \epsilon^{\prime}(\epsilon)=0$, so that the following holds. Suppose that $h$ is a Riemannian metric on $N=T^{3}$ with $\operatorname{diam}(N, h)=1$ and $\max _{p \in N}\left|\operatorname{Rm}_{h}\right|(p) \leq \epsilon$. Then there is a finite cover $\widehat{N}$ of $N$ so that $(\widehat{N}, \widehat{h})$ has distance at most $\epsilon^{\prime}(\epsilon)$ in the $C^{1}$-topology from an element of $K$.

Proof. Given $i_{0}, D_{1}>0$ and $D_{2}<\infty$, let $K_{i_{0}, D_{1}, D_{2}}$ denote the isometry classes of flat Riemannian metrics on $T^{3}$ with diameter in $\left[D_{1}, D_{2}\right]$ and injectivity radius bounded below by $i_{0}$. For any $\alpha \in \mathbb{Z}^{+}$, the set $K_{i_{0}, D_{1}, D_{2}}$ is compact in the $C^{\alpha}$-topology. We will take $K=K_{i_{0}, D_{1}, D_{2}}$, where the parameters $i_{0}, D_{1}$ and $D_{2}$ will be determined in the proof.

Given $K$, suppose that the claim is false. Then there is some $\epsilon^{\prime}>0$ along with a sequence $\left\{\left(N_{i}, h_{i}\right)\right\}_{i=1}^{\infty}$ of Riemannian 3-tori with $\operatorname{diam}\left(N_{i}, h_{i}\right)=$ 1 and $\max _{p \in N_{i}}\left|\operatorname{Rm}_{g_{i}}\right|(p) \leq \frac{1}{i}$, but with the property that for no $i$ is there a finite cover $\left(\widehat{N}_{i}, \widehat{h}_{i}\right)$ of $\left(N_{i}, h_{i}\right)$ with distance less than $\epsilon^{\prime}$ in the $C^{1}$-topology from an element of $K$. Let $\widetilde{N}_{i}$ be the universal cover of $N_{i}$. Pick $p_{i} \in N_{i}$ and let $\widetilde{p}_{i}$ be a lift to $\widetilde{N}_{i}$. As $i \rightarrow \infty,\left(\widetilde{N}_{i}, \widetilde{p}_{i}\right)$ converges to $\left(\mathbb{R}^{3}, 0\right)$ in the pointed $C^{1}$-topology; this follows from a uniform lower bound on the injectivity radius at $\widetilde{p}_{i}[$ Ron07, (3.2)] and $C^{1}$-convergence results. Let $r_{1, i} \in \pi_{1}\left(N_{i}, p_{i}\right)$ be a nontrivial element which minimizes $d\left(\widetilde{p}_{i}, r_{1, i} \widetilde{p}_{i}\right)$. Let $r_{2, i} \in \pi_{1}\left(N_{i}, p_{i}\right)-\left\langle r_{1, i}\right\rangle$ minimize $d\left(\widetilde{p}_{i}, r_{2, i} \widetilde{p}_{i}\right)$. Let $r_{3, i} \in \pi_{1}\left(N_{i}, p_{i}\right)-\left\langle r_{1, i}, r_{2, i}\right\rangle$ minimize $d\left(\widetilde{p}_{i}, r_{3, i} \widetilde{p}_{i}\right)$. By the diameter condition, for large $i$ we have $d\left(\widetilde{p}_{i}, r_{3, i} \widetilde{p}_{i}\right) \leq 10$.

Let $\gamma_{1, i}, \gamma_{2, i}, \gamma_{3, i}$ be minimizing geodesics from $\widetilde{p}$ to $r_{1, i} \widetilde{p}, r_{2, i} \widetilde{p}, r_{3, i} \widetilde{p}$. There is a universal constant $c>0$ so that for each $i$, pairs from $\left\{\gamma_{1, i}^{\prime}(0), \gamma_{2, i}^{\prime}(0), \gamma_{3, i}^{\prime}(0)\right\}$ form an angle at $\widetilde{p}$ which is at least $c$ Gro78, Section 2.3]. Let $k_{1, i}$ and $k_{2, i}$ be positive integers so that $k_{1, i} d\left(\widetilde{p}_{i}, r_{1, i} \widetilde{p}_{i}\right)$ and $k_{2, i} d\left(\widetilde{p}_{2}, r_{2, i} \widetilde{p}_{i}\right)$ lie in $\left[\frac{1}{10}, 10\right]$. Let $\widehat{N}_{i}$ be the cover of $N_{i}$ so that 
RICCI FLOW ON THREE-DIMENSIONAL MANIFOLDS WITH SYMMETRY 19 $\pi_{1}\left(\widehat{N}_{i}, \widehat{p}_{i}\right)$ has generators $r_{1, i}^{k_{1, i}}, r_{2, i}^{k_{2, i}}, r_{3, i}$. After passing to a subsequence, we can assume that as $i \rightarrow \infty$, with respect to the approximations of $\widetilde{N}_{i}$ by $\mathbb{R}^{3}$, the triples $r_{1, i}^{k_{1, i}} \widetilde{p}_{i}, r_{2, i}^{k_{2, i}} \widetilde{p}_{i}, r_{3, i} \widetilde{p}_{i}$ converge to vectors $v_{1}, v_{2}, v_{3}$ in $\mathbb{R}^{3}$ with lengths in $\left[\frac{1}{10}, 10\right]$ and mutual angles at least $c$. Then $\lim _{i \rightarrow \infty}\left(\widehat{N}_{i}, \widehat{h}_{i}\right)=\mathbb{R}^{3} /\left(\mathbb{Z} v_{1}+\mathbb{Z} v_{2}+\mathbb{Z} v_{3}\right)$ in the $C^{1}$-topology. We can find $i_{0}, D_{1}>0$ and $D_{2}<\infty$, computed in terms of $c$, so that $\mathbb{R}^{3} /\left(\mathbb{Z} v_{1}+\mathbb{Z} v_{2}+\mathbb{Z} v_{3}\right)$ has diameter in $\left[D_{1}, D_{2}\right]$ and injectivity radius bounded below by $i_{0}$. This contradicts the properties of the sequence $\left\{\left(N_{i}, h_{i}\right)\right\}_{i=1}^{\infty}$. The claim follows.

By (2.63), for any $\epsilon^{\prime}>0$, if $t$ is large enough then we can apply Claim 2.16 to $(N, h(t))$. Using the higher derivative curvature estimates coming from the Ricci flow and applying a similar argument as in the proof of Claim 2.16, for any $\alpha \in \mathbb{Z}^{+}$we can also say that there is a compact subset $K_{\alpha}$ of the moduli space of flat metrics on $T^{3}$ so that for any $\epsilon^{\prime}>0$, if $t$ is large enough then a finite cover $(\widehat{N}, \widehat{h}(t))$ is $\epsilon^{\prime}$-close in the $C^{\alpha}$-topology to an element of $K_{\alpha}$. Taking $\alpha$ large, we can now apply [GIK02, Theorem 3.7] to conclude that as $t \rightarrow \infty,(\widehat{N}, \widehat{h}(t))$ (or more precisely the solution of (2.7)) converges to a flat metric on $T^{3}$. Furthermore, the convergence is exponentially fast. (Strictly speaking, GIK02 considers the evolution of a Riemannian metric $h$ which is sufficiently close to a fixed flat metric, but the arguments clearly extend to the setting of closeness to a compact set $K_{\alpha}$ of flat metrics, since there will be uniform control on the constants.) Because of the equivariance of Ricci flow under isometries, the same is true for $(N, h(t))$.

This proves Proposition 2.9.

2.6. Negative Euler characteristic. By Proposition 2.8, the flow (2.8) or, equivalently, (2.7) exists forever. We start with the following proposition.

Proposition 2.17. We have $\max _{p \in N}\left|\mathrm{Rm}^{N}\right|(p, t)=O\left(t^{-1}\right)$.

Proof. The argument is similar to that in the proof of Proposition 2.10 . If the statement were not true, i.e. if $\limsup _{t \rightarrow \infty} t \cdot \max _{m \in M}\left|\operatorname{Rm}^{N}\right|(m, t)=$ $\infty$, then we begin the argument exactly the same as in the proof of Proposition 2.10 to take a limit of rescalings at times $\left\{t_{i}\right\}_{i=1}^{\infty}$. This limit is an eternal solution of the form $h_{\infty}(t)=g_{\infty}(t)+C d \theta^{2}$, where $C$ is a constant and $g_{\infty}(t)$ is a nonflat eternal solution to the Ricci flow on a two-dimensional manifold $M_{\infty}$, with complete time slices and nonnegative bounded curvature. As in the proof of Proposition 2.10, 
$\left(M_{\infty}, g_{\infty}(\cdot)\right)$ must be a cigar soliton and

$$
\int_{M_{\infty}} R_{g_{\infty}(0)} d V_{g_{\infty}(0)}=4 \pi
$$

For large $i$, there is a bounded domain $S_{i} \subset\left(M, g\left(t_{i}\right)\right)$ which, after rescaling, is almost isometric to a large region in the cigar soliton. Then

$$
\int_{S_{i}} R_{g\left(t_{i}\right)} d V_{g\left(t_{i}\right)} \geq 3 \pi
$$

Furthermore, by (2.21) and Corollary 2.3, we have

$$
\int_{M-S_{i}} R_{g\left(t_{i}\right)} d V_{g\left(t_{i}\right)} \geq-\frac{1}{t_{i}} \operatorname{Vol}_{g\left(t_{i}\right)}\left(M-S_{i}\right) \geq-\frac{V\left(t_{i}\right)}{t_{i}} \geq 4 \pi \chi(M) \cdot\left(1+o\left(i^{0}\right)\right) .
$$

Adding (2.65) and (2.66), and taking $i \rightarrow \infty$, contradicts the GaussBonnet theorem for $M$. tion.

Put $\widehat{g}(t)=\frac{g(t)}{t}$. Let $\widehat{\nabla}$ denote the corresponding Levi-Civita connec-

Claim 2.18. There exist $i_{0}>0$ and $t_{0}>0$ so that for every $t \geq t_{0}$, there is a point $m_{t} \in M$ where the injectivity radius satisfies $\operatorname{inj}_{\widehat{g}(t)}\left(m_{t}\right) \geq$ $i_{0}$.

Proof. By (2.2) and Proposition 2.17, for $t \geq 1$ the metrics $(M, \widehat{g}(t))$ have uniformly bounded curvature. If the claim were not true then for every $\epsilon>0$, there would be some $t_{\epsilon} \geq 1$ so that $\operatorname{inj}_{\widehat{g}\left(t_{\epsilon}\right)}(m)<\epsilon$ for all $m \in M$. Then $M$ would have an F-structure and hence a vanishing Euler characteristic [CG90]. This is a contradiction.

Proposition 2.19. For any $i_{0}>0$, define the $i_{0}$-thick part of $(M, \widehat{g}(t))$ by

$$
X_{i_{0}}(t)=\left\{m \in M: \operatorname{inj}_{\widehat{g}(t)}(m) \geq i_{0}\right\}
$$

Then

$$
\lim _{t \rightarrow \infty} \sup _{x \in X_{i_{0}}(t)}\left|R_{\widehat{g}(t)}(x)+1\right|=0
$$

and

$$
\lim _{t \rightarrow \infty} \sup _{x \in X_{i_{0}}(t)}|\widehat{\nabla} u|_{\widehat{g}(t)}(x)=0 .
$$

Proof. Suppose that the proposition is not true. Then there are some $i_{0}, \epsilon>0$ along with sequences $t_{i} \rightarrow \infty$ and $\left\{m_{i}\right\}_{i=1}^{\infty}$ so that for each $i$, $\operatorname{inj}_{\widehat{g}\left(t_{i}\right)}\left(m_{i}\right) \geq i_{0}$ and either $\left|R_{\widehat{g}\left(t_{i}\right)}\left(m_{i}\right)+1\right| \geq \epsilon$ or $|\widehat{\nabla} u|_{\widehat{g}\left(t_{i}\right)}\left(m_{i}\right) \geq \epsilon$. 
RICCI FLOW ON THREE-DIMENSIONAL MANIFOLDS WITH SYMMETRY 21

By Hamilton's compactness theorem and the derivative estimates on $u$ from [Lis08, Section 5], after passing to a subsequence we can assume that there is a smooth pointed limit of flows

$$
\lim _{i \rightarrow \infty}\left(M, m_{i}, \frac{1}{t_{i}} g\left(t_{i} t\right), u\left(t_{i} t\right)\right)=\left(M_{\infty}, m_{\infty}, g_{\infty}(t), u_{\infty}(t)\right) .
$$

For any bounded domain $S \subset M_{\infty}$, using Corollary 2.2 we have that at any time $a$,

$$
\int_{S}\left|\nabla u_{\infty}\right|_{g_{\infty}(a)}^{2} d V_{g_{\infty}(a)} \leq \limsup _{i \rightarrow \infty} \int_{M}\left|\nabla u\left(t_{i} a\right)\right|_{g\left(t_{i} a\right)}^{2} d V_{g\left(t_{i} a\right)}=0
$$

Thus $u_{\infty}$ is spatially constant at time $a$. Because $a$ is arbitrary, and $u_{\infty}$ satisfies the time-dependent heat equation, it follows that $u_{\infty}$ is also temporally constant. In particular,

$$
0=\left|\nabla u_{\infty}\right|_{g_{\infty}(1)}\left(m_{\infty}\right)=\lim _{i \rightarrow \infty}|\widehat{\nabla} u|_{\widehat{g}\left(t_{i}\right)}\left(m_{i}\right)
$$

Now $R_{g_{\infty}(t)}+\frac{1}{t} \geq 0$. Given $0<a<b<\infty$, equations (2.35) and (2.72), along with Corollary 2.3, give

$$
\begin{aligned}
& \int_{a}^{b} \int_{M_{\infty}}\left(R_{g_{\infty}(t)}+\frac{1}{t}\right) d V_{g_{\infty}(t)} \frac{d t}{t}= \\
& \int_{a}^{b} \int_{M_{\infty}}\left(R_{g_{\infty}(t)}-\left|\nabla u_{\infty}\right|^{2}(t)+\frac{1}{t}\right) d V_{g_{\infty}(t)} \frac{d t}{t} \\
& \leq \lim _{i \rightarrow \infty} \int_{t_{i} a}^{t_{i} b} \int_{M}\left(R_{g(t)}-|\nabla u|^{2}(t)+\frac{1}{t}\right) d V_{g(t)} \frac{d t}{t} \\
& =\lim _{i \rightarrow \infty}\left(\frac{V\left(t_{i} a\right)}{t_{i} a}-\frac{V\left(t_{i} b\right)}{t_{i} b}\right)=0 .
\end{aligned}
$$

Since $a$ and $b$ were arbitrary, we obtain $R_{g_{\infty}(t)}(m)=-\frac{1}{t}$ for all $m \in M_{\infty}$ and $t \in(0, \infty)$. In particular,

$$
-1=R_{g_{\infty}(1)}\left(m_{\infty}\right)=\lim _{i \rightarrow \infty} R_{\widehat{g}\left(t_{i}\right)}\left(m_{i}\right) .
$$

Equations (2.72) and (2.74) together contradict our assumptions about $\left\{t_{i}\right\}_{i=1}^{\infty}$ and $\left\{m_{i}\right\}_{i=1}^{\infty}$, thereby proving the proposition.

Remark 2.20. In the case $\chi(M)<0$ one could hope for a bound $\operatorname{diam}(M, g(t))=O(\sqrt{t})$. With such a bound one could conclude that in Proposition 2.19, there is some $i_{0}>0$ so that for large $t, X_{i_{0}}(t)$ is all of $M$. Without such a bound, one could imagine that as $t \rightarrow \infty$, the manifolds $(M, \widehat{g}(t))$ approach a family of surfaces of constant curvature $-\frac{1}{2}$ that slowly pinch off a closed geodesic. 


\section{TORUS BUndLeS}

In this section we prove Theorem 1.6. In Subsection 3.1 we write down the Ricci flow equations with a $U(1) \times U(1)$ symmetry and give some direct consequences. In Subsection 3.2 we show that the Ricci flow exists for all $t \in[0, \infty)$. In Subsection 3.3 we prove that the curvature decays like $O\left(t^{-1}\right)$. In Subsection 3.4 we show that the length of the

circle base is $O(\sqrt{t})$. In Subsection 3.5 we finish the proof of Theorem 1.6.

3.1. Twisted principal $U(1) \times U(1)$ bundles. Let $N$ be a an orientable 3-manifold which is the total space of a fiber bundle $\pi: N \rightarrow$ $S^{1}$, with $T^{2}$-fibers. Choosing an orientation of $S^{1}$, the fiber bundle has a holonomy $H \in \mathrm{SL}(2, \mathbb{Z})=\pi_{0}\left(\operatorname{Diff}^{+}\left(T^{2}\right)\right)$. Taken up to inverses, $H$ determines the topological type of the fiber bundle. We refer to [Sco83, Theorem 5.5] for the Thurston types of such fiber bundles. If $H$ is elliptic, i.e. has finite order, then $N$ has a flat structure. If $H$ is parabolic, i.e. $|\operatorname{Tr}(H)| \leq 2$ but $H$ is not elliptic, then $N$ has a Nil structure. If $H$ is hyperbolic, i.e. has no eigenvalues on the unit circle, then $N$ has a Sol structure.

If $H$ is the identity then $N=S^{1} \times T^{2}$ is the total space of a principal $U(1) \times U(1)$ bundle. That is, $N$ admits a free $U(1) \times U(1)$ action. In general, $N$ is the total space of a twisted principal $U(1) \times U(1)$ bundle, where "twisted" refers to the fact that $H$ may be nontrivial. The setup is a special case of that in [Lot10, Section 4.1]. Let $\mathcal{E}$ be a local system over $S^{1}$ of groups isomorphic to $U(1) \times U(1)$. We assume that the holonomy of the local system is $H \in \operatorname{Aut}(U(1) \times U(1))$. Then there is a notion of a free $\mathcal{E}$-action on the $T^{2}$-bundle $N$, which generalizes the global $U(1) \times U(1)$ action that exists when $H$ is the identity.

Let $h$ be a Riemannian metric on $N$ which is $\mathcal{E}$-invariant. There is a corresponding horizontal distribution $\mathcal{H}$ on $N$. Since $\mathcal{H}$ is onedimensional, it is integrable. Note that even if $H$ is the identity, $\mathcal{H}$ can have a nontrivial holonomy in $U(1) \times U(1)$, when going around the circle base. Thus the flat structure on $\mathcal{E}$ is logically distinct from the flat structure on $N$ coming from $\mathcal{H}$.

Let $V$ be a coordinate chart of $S^{1}$, with local coordinate $y$. The integrability of $\mathcal{H}$ gives a local trivialization $V \times T^{2}$ of $\pi^{-1}(V)$. The restriction of $h$ to $\pi^{-1}(V)$ is invariant under the $U(1) \times U(1)$ action coming from $\left.\mathcal{E}\right|_{V}$. Using this action and the local trivialization, let $x_{1}, x_{2}$ denote local angular coordinates on the $T^{2}$-fibers. In terms of 

these coordinates we can write

$$
h=\sum_{i, j=1}^{2} G_{i j}(y) d x^{i} d x^{j}+g_{y y}(y) d y^{2} .
$$

We use $i, j, k, l$ for vertical indices. From [Lot10, Section 4.2], nonzero components of the curvature tensor of $(N, h)$ are

$$
\begin{aligned}
& R_{i j k l}^{N}=-\frac{1}{4} g^{y y} G_{i k, y} G_{j l, y}+\frac{1}{4} g^{y y} G_{i l, y} G_{j k, y}, \\
& R_{i y j y}^{N}=-\frac{1}{2} G_{i j ; y y}+\frac{1}{4} G^{k l} G_{i k, y} G_{j l, y},
\end{aligned}
$$

where

$$
G_{i j ; y y}=G_{i j, y y}-\Gamma_{y y}^{y} G_{i j, y}=G_{i j, y y}-\frac{1}{2} \frac{g_{y y, y}}{g_{y y}} G_{i j, y} .
$$

The nonzero components of the Ricci tensor are

$$
\begin{aligned}
R_{i j} & =-\frac{1}{2} g^{y y} G_{i j ; y y}-\frac{1}{4} g^{y y} G^{k l} G_{k l, y} G_{i j, y}+g^{y y} \frac{1}{2} G^{k l} G_{i k, y} G_{l j, y}, \\
R_{y y} & =-\frac{1}{2} G^{i j} G_{i j ; y y}+\frac{1}{4} G^{i j} G_{j k, y} G^{k l} G_{l i, y} .
\end{aligned}
$$

The scalar curvature is

$$
R=-g^{y y} G^{i j} G_{i j ; y y}+\frac{3}{4} g^{y y} G^{i j} G_{j k, y} G^{k l} G_{l i, y}-\frac{1}{4} g^{y y} G^{i j} G_{i j, y} G^{k l} G_{k l, y} .
$$

We will use matrix notation $G=\left(G_{i j}\right)$. Note that $G$ is symmetric and positive-definite. The Ricci flow equation

$$
\frac{d h}{d t}=-2 \operatorname{Ric}_{h(t)}
$$

preserves the local $U(1) \times U(1)$ invariance of the metric. In terms of $g$ and $G$, it becomes

$$
\begin{aligned}
\frac{\partial g_{y y}}{\partial t} & =\operatorname{Tr}\left(G^{-1} G_{; y y}\right)-\frac{1}{2} \operatorname{Tr}\left(\left(G^{-1} G_{, y}\right)^{2}\right) \\
\frac{\partial G}{\partial t} & =g^{y y} G_{; y y}+\frac{1}{2} g^{y y} \operatorname{Tr}\left(G^{-1} G_{, y}\right) G_{, y}-g^{y y} G_{, y} G^{-1} G_{, y} .
\end{aligned}
$$

Adding a Lie derivative with respect to $-\nabla \ln \sqrt{\operatorname{det}(G)}$ to the righthand side gives the modified equations

$$
\begin{aligned}
\frac{\partial g_{y y}}{\partial t} & =\frac{1}{2} \operatorname{Tr}\left(\left(G^{-1} G_{, y}\right)^{2}\right), \\
\frac{\partial G}{\partial t} & =g^{y y}\left(G_{; y y}-G_{, y} G^{-1} G_{, y}\right) .
\end{aligned}
$$


Taking $y$ to run over $\mathbb{R}$, the periodicity condition on $G$ is

$$
G(y+1, t)=H^{T} G(y, t) H .
$$

The manifold $N$ can be recovered by taking the quotient of $\mathbb{R} \times T^{2}$ by the equivalence relation $(y+1, x) \sim(y, H x+b)$, where $b$ is some fixed element of $\mathbb{R}^{2} / \mathbb{Z}^{2}$.

Hereafter we will mainly work with (3.8). An example of a solution to $(3.8)$ is

$$
\begin{aligned}
g_{y y}(y, t) & =4 c^{2}(t+a), \\
G(y, t) & =\left(\begin{array}{cc}
e^{2 c y} & 0 \\
0 & e^{-2 c y}
\end{array}\right) .
\end{aligned}
$$

If we take $y$ to be defined in $\mathbb{R} / \mathbb{Z}$ then we get a metric on a bundle with hyperbolic holonomy $H \in \mathrm{SL}(2, \mathbb{Z})$, provided that $H$ has eigenvalues $e^{c}$ and $e^{-c}$. Here $\partial_{x^{1}}$ and $\partial_{x^{2}}$ are corresponding eigenvectors. The length of the circle base is $2 c \sqrt{t+a}$.

Given $s>0$ and a solution $(g(\cdot), G(\cdot))$ of (3.8), we obtain another solution of (3.8) by putting

$$
\begin{aligned}
g_{s}(t) & =\frac{1}{s} g(s t), \\
G_{s}(t) & =G(s t) .
\end{aligned}
$$

Put

$$
h_{s}=(d x)^{T} G_{s} d x+g_{s}
$$

Then

$$
\left|\mathrm{Rm}_{h_{s}}\right|^{2}=s^{2}\left|\mathrm{Rm}_{h}\right|^{2}
$$

\section{Lemma 3.1.}

$$
\frac{\partial \ln \operatorname{det}(G)}{\partial t}=\triangle \ln \operatorname{det}(G)
$$

Proof. We have

$$
\begin{aligned}
\frac{\partial \ln \operatorname{det}(G)}{\partial t} & =\operatorname{Tr}\left(G^{-1} \frac{\partial G}{\partial t}\right) \\
& =g^{y y} \operatorname{Tr}\left(G^{-1}\left(G_{; y y}-G_{, y} G^{-1} G_{, y}\right)\right) .
\end{aligned}
$$

Passing to an arc-length parameter $s$ on $S^{1}$ at a given time $t$, we obtain

$$
\frac{\partial \ln \operatorname{det}(G)}{\partial t}=\operatorname{Tr}\left(G^{-1}\left(G_{, s s}-G_{, s} G^{-1} G_{, s}\right)\right) \text {. }
$$


RICCI FLOW ON THREE-DIMENSIONAL MANIFOLDS WITH SYMMETRY 25

On the other hand

$$
\begin{aligned}
\triangle \ln \operatorname{det}(G) & =\frac{d^{2}}{d s^{2}} \ln \operatorname{det}(G)=\frac{d}{d s} \operatorname{Tr}\left(G^{-1} G_{, s}\right) \\
& =\operatorname{Tr}\left(G^{-1} G_{, s s}-G^{-1} G_{, s} G^{-1} G_{, s}\right) .
\end{aligned}
$$

This proves the lemma.

Note that $\operatorname{det}(G)$ is globally defined on $S^{1}$ since the holonomy lies in $\operatorname{SL}(2, \mathbb{Z})$. It represents the squares of the volumes of the fibers.

Corollary 3.2. There are constants $C_{1}, C_{2}>0$ so that for all $y \in S^{1}$ and all times $t$ for which the flow exists.

$$
C_{1} \leq \sqrt{\operatorname{det}(G)}(y, t) \leq C_{2} .
$$

Proof. This follows from applying the maximum principle to Lemma 3.1.

Lemma 3.3. Put

$$
\mathcal{E}=g^{y y} \operatorname{Tr}\left(\left(G^{-1} G_{, y}\right)^{2}\right)
$$

Then

$$
\frac{\partial \mathcal{E}}{\partial t}=\triangle \mathcal{E}-\frac{\mathcal{E}^{2}}{2}-2 g^{y y} g^{y y} \operatorname{Tr}\left(\left(G^{-1} G_{; y y}-\left(G^{-1} G_{, y}\right)^{2}\right)^{2}\right) .
$$

Proof. Differentiating (3.19) with respect to $t$ gives

$$
\begin{aligned}
\frac{\partial \mathcal{E}}{\partial t}= & -\frac{1}{2} g^{y y} g^{y y}\left(\operatorname{Tr}\left(\left(G^{-1} G_{, y}\right)^{2}\right)\right)^{2} \\
& -2 g^{y y} \operatorname{Tr}\left(G^{-1} G_{, y} G^{-1} g^{y y}\left(G_{; y y}-G_{, y} G^{-1} G_{, y}\right) G^{-1} G_{, y}\right) \\
& +2 g^{y y} \operatorname{Tr}\left(G^{-1} G_{, y} G^{-1}\left(g^{y y}\left(G_{; y y}-G_{, y} G^{-1} G_{, y}\right)\right)_{, y}\right) .
\end{aligned}
$$

Switching to an arc-length parameter $s$ at a given time $t$, we obtain

$$
\begin{aligned}
\frac{\partial \mathcal{E}}{\partial t}= & -\frac{\mathcal{E}^{2}}{2}-2 \operatorname{Tr}\left(G^{-1} G_{, s} G^{-1}\left(G_{, s s}-G_{, s} G^{-1} G_{, s}\right) G^{-1} G_{, s}\right) \\
& +2 \operatorname{Tr}\left(G^{-1} G_{, s} G^{-1}\left(G_{, s s}-G_{, s} G^{-1} G_{, s}\right)_{, s}\right) .
\end{aligned}
$$

One now computes that

$$
\begin{aligned}
\frac{\partial \mathcal{E}}{\partial t}= & -\frac{\mathcal{E}^{2}}{2}+\left(\operatorname{Tr}\left(\left(G^{-1} G_{, s}\right)^{2}\right)\right)_{, s s} \\
& -2 \operatorname{Tr}\left(\left(G^{-1} G_{, s s}-\left(G^{-1} G_{, s}\right)^{2}\right)^{2}\right) .
\end{aligned}
$$

This proves the lemma. 
Note that

$$
\mathcal{E}=g^{y y} \operatorname{Tr}\left(\left(G^{-\frac{1}{2}} G_{, y} G^{-\frac{1}{2}}\right)^{2}\right)
$$

is nonnegative, since $G^{-\frac{1}{2}} G_{, y} G^{-\frac{1}{2}}$ is symmetric.

Corollary 3.4. For all $y \in S^{1}$ and all $t \geq 0$ for which the flow exists, we have $\mathcal{E}(y, t) \leq \frac{2}{t}$.

Proof. We have

$$
\begin{aligned}
& \operatorname{Tr}\left(\left(G^{-1} G_{; y y}-\left(G^{-1} G_{, y}\right)^{2}\right)^{2}\right)= \\
& \operatorname{Tr}\left(\left(G^{-\frac{1}{2}} G_{; y y} G^{-\frac{1}{2}}-\left(G^{-\frac{1}{2}} G_{, y} G^{-\frac{1}{2}}\right)^{2}\right)^{2}\right),
\end{aligned}
$$

which is nonnegative since $G^{-\frac{1}{2}} G_{; y y} G^{-\frac{1}{2}}-\left(G^{-\frac{1}{2}} G_{, y} G^{-\frac{1}{2}}\right)^{2}$ is symmetric. The corollary now follows from applying the maximum principle to Lemma 3.3 .

\subsection{Nonsingularity of the flow.}

Proposition 3.5. Given $h(0)$ as described in Subsection 3.1, the flow (3.8) exists for $t \in[0, \infty)$.

Proof. If not then there is a singularity at some time $T<\infty$. Specializing the modified $W$-functional of [Lot10, Definition 4.48] to our case, it becomes

$$
\begin{aligned}
& W(G, g, f, \tau)= \\
& \int_{S^{1}}\left[\tau\left(|\nabla f|^{2}-\frac{1}{4} g^{y y} \operatorname{Tr}\left(\left(G^{-1} G_{, y}\right)^{2}\right)\right)+f-1\right](4 \pi \tau)^{-\frac{1}{2}} e^{-f} \sqrt{g_{y y}} d y .
\end{aligned}
$$

Using this modified $W$-functional, we can go through the same steps as in the proof of [Lis08, Theorem 7.9] to conclude that there is a blowup limit $\left(M_{\infty}, m_{\infty}, g_{\infty}(\cdot), G_{\infty}(\cdot)\right)$ where

- $G_{\infty}=$ const. and

- $g_{\infty}$ is a nonflat Ricci flow solution on the 1-manifold $M_{\infty}$.

However, there is no such nonflat Ricci flow solution. This proves the proposition. 
RICCI FLOW ON THREE-DIMENSIONAL MANIFOLDS WITH SYMMETRY 27

\subsection{Curvature bound.}

Proposition 3.6. We have $\max _{p \in N}\left|\mathrm{Rm}^{N}\right|(p, t)=O\left(t^{-1}\right)$.

Proof. Suppose that the proposition is false. We take a rescaling limit as in the proof of Proposition 2.10 to obtain a nonflat eternal solution $\left(M_{\infty}, p_{\infty}, g_{\infty}(\cdot), G_{\infty}(\cdot)\right)$ on a one-dimensional étale groupoid $M_{\infty}$. (It will follow from Lemma 3.12 that $M_{\infty}$ is a one-dimensional manifold.) From Corollary [3.4, $G_{\infty}$ is constant. Then $g_{\infty}$ is a nonflat Ricci flow solution on a one-dimensional space, which is a contradiction.

3.4. Diameter bound. We compute how the length of the base circle varies with time.

Lemma 3.7. Put $L(t)=\int_{S^{1}} \sqrt{g_{y y}}(y) d y$. Then

$$
\frac{d L}{d t}=\frac{1}{4} \int_{S^{1}} \mathcal{E}(y, t) \sqrt{g_{y y}}(y) d y \text {. }
$$

Proof. We have

$$
\frac{d L}{d t}=\frac{1}{2} \int_{S^{1}} g^{y y} \frac{\partial g_{y y}}{\partial t} \sqrt{g_{y y}}(y) d y=\frac{1}{4} \int_{S^{1}} g^{y y} \operatorname{Tr}\left(\left(G^{-1} G_{, y}\right)^{2}\right) \sqrt{g_{y y}}(y) d y .
$$

This proves the lemma.

In particular, $L(t)$ is monotonically nondecreasing in $t$.

Lemma 3.8. $t^{-\frac{1}{2}} L(t)$ is monotonically nonincreasing in $t$.

Proof. From Corollary 3.4 and Lemma 3.7, we derive

$$
\frac{d L}{d t} \leq \frac{L(t)}{2 t}
$$

The lemma follows.

\subsection{Long-time behavior.}

Lemma 3.9. Given $x \in T^{2}$ and linearly independent vectors $v_{1}, v_{2} \in$ $T_{x} T^{2}$, there is a constant $C\left(v_{1}, v_{2}\right)<\infty$ with the following property. Suppose that $g_{T^{2}}$ is a flat metric on $T^{2}$. Then

$$
\operatorname{diam}\left(T^{2}, g_{T^{2}}\right) \leq C\left(v_{1}, v_{2}\right)\left(\left|v_{1}\right|_{T_{T^{2}}}+\left|v_{2}\right|_{g_{T^{2}}}\right) .
$$

Proof. Let $\mathcal{V}_{1}$ and $\mathcal{V}_{2}$ be the affine-parallel vector fields on $T^{2}$ that extend $v_{1}$ and $v_{2}$, respectively. Let $g_{0}$ be a fixed flat metric on $T^{2}$. There is some $c<\infty$ so that any pair of points in $T^{2}$ can be joined by flowing first in the $\mathcal{V}_{1}$-direction for a length at most $c$ and then flowing in the $\mathcal{V}_{2}$-direction for a length at most $c$. With respect to $g_{T^{2}}$, the 
length of this path is bounded above by $c \frac{\left|v_{1}\right|_{g_{T}}}{\left|v_{1}\right| g_{0}}+c \frac{\left|v_{2}\right|_{g_{T}}}{\left|v_{2}\right|_{g_{0}}}$. The lemma follows.

We use the notation in the statement of Theorem 1.6.

3.5.1. Elliptic holonomy. If $H$ is elliptic then after pulling back the $T^{2}$-bundle from a finite covering $S^{1} \rightarrow S^{1}$, we can assume that $H=I$. Then $G(y, t)$ is a globally defined matrix-valued function of $y \in \mathbb{R} / \mathbb{Z}$ and $t \in[0, \infty)$.

Lemma 3.10. The intrinsic diameter of the $T^{2}$-fibers is uniformly bounded above in $t$.

Proof. Given $v \in \mathbb{R}^{2}$, equation (3.8) implies that

$$
\frac{\partial}{\partial t}\langle v, G v\rangle=g^{y y}\langle v, G v\rangle_{; y y}-g^{y y}\left\langle v, G_{, y} G^{-1} G_{, y} v\right\rangle \text {. }
$$

The maximum principle now implies that $\langle v, G(y, t) v\rangle$ is uniformly bounded above for $y \in S^{1}$ and $t \in[0, \infty)$. The lemma follows from Lemma 3.9.

Proposition 3.11. $\operatorname{diam}(N, h(t))=O(\sqrt{t})$.

Proof. This follows from Lemmas 2.13, 3.8 and 3.10.

From [Lot10, Theorem 1.2.1],

$$
\left(\max _{p \in N}\left|\operatorname{Rm}_{h}^{N}\right|(p, t)\right) \cdot \operatorname{diam}^{2}(N, h(t))=o(t) .
$$

The argument of Subsection 2.5 now shows that $\lim _{t \rightarrow \infty} h(t)$ exists and is a flat metric on $N$, with the convergence being exponentially fast.

\subsubsection{Hyperbolic holonomy. Suppose that $H$ is hyperbolic.}

Let $P(2, \mathbb{R})$ denote the positive-definite symmetric $2 \times 2$ matrices. Given $G \in P(2, \mathbb{R})$ and symmetric $2 \times 2$ matrices $\delta_{1} G, \delta_{2} G \in T_{G} P(2, \mathbb{R})$, we define their inner product by

$$
\left\langle\delta_{1} G, \delta_{2} G\right\rangle=\frac{1}{2} \operatorname{Tr}\left(G^{-1}\left(\delta_{1} G\right) G^{-1}\left(\delta_{2} G\right)\right) .
$$

Consider the map $\Phi: \mathbb{R} \times \mathrm{SL}(2, \mathbb{R}) \rightarrow P(2, \mathbb{R})$ given by $(u, M) \rightarrow$ $e^{u} M^{T} M$. Identifying $\mathrm{SO}(2) \backslash \mathrm{SL}(2, \mathbb{R})$ with the hyperbolic space $H^{2}$, the map $\Phi$ passes to an isometry $\mathbb{R} \times H^{2} \rightarrow P(2, \mathbb{R})$.

With respect to this isometry, the action of $\mathrm{SL}(2, \mathbb{Z})$ on $P(2, \mathbb{R})$ (by $\left.(A, G) \rightarrow A^{T} G A\right)$ becomes the product of the trivial action of $\operatorname{SL}(2, \mathbb{Z})$ on $\mathbb{R}$ with the isometric action of $\operatorname{SL}(2, \mathbb{Z})$ on $H^{2}$. Letting $\langle H\rangle$ denote the cyclic subgroup of $\mathrm{SL}(2, \mathbb{Z})$ generated by the holonomy $H$, equation (3.9) can be interpreted as saying that for each $t$, the function $G(\cdot, y)$ 
describes a smooth map $S^{1} \rightarrow\left(\mathbb{R} \times H^{2} /\langle H\rangle\right)$ whose homotopy class is specified by $H$.

Lemma 3.12. There is a constant $c>0$ so that for all $t \in[0, \infty)$, $L(t) \geq c \sqrt{t}$.

Proof. Lemma 3.4 says that the Lipschitz constant of the map $G(\cdot, t)$ from $[0,1]$ to $P(2, \mathbb{R})$ is bounded above by $\frac{1}{\sqrt{t}}$. As $H$ is hyperbolic, there is a minimal length $c>0$ among all noncontractible closed curves in $H^{2} /\langle H\rangle$. Consequently, the distance between $G(0, t)$ and $G(1, t)=$ $H^{T} G(0, t) H$ in $P(2, \mathbb{R})$ is bounded below by $c$. Thus $L(t) \geq c \sqrt{t}$.

Recall the definitions of $g_{s}$ and $G_{s}$ from (3.11). By an appropriate $s$-dependent choice of basis for $\mathbb{R}^{2}$, we can assume that $G_{s}(0,1)=I$. In making such a choice of basis, we are ignoring the lattice structure that comes from writing $T^{2}$ as a quotient of $\mathbb{R}^{2}$. We are simply treating $G$ and $g$ as functions which satisfy (3.8) and (3.9).

Let $X$ be the real symmetric matrix so that $e^{X}=H^{T} H$.

Proposition 3.13. For any sequence $\left\{s_{j}\right\}_{j=1}^{\infty}$ going to infinity, after passing to a subsequence and possibly reparametrizing $S^{1}$, we have

$$
\lim _{j \rightarrow \infty} g_{s_{j}}(y, t)=\frac{t}{2} \operatorname{Tr}\left(X^{2}\right) d y^{2}
$$

and

$$
\lim _{j \rightarrow \infty} G_{s_{j}}(y, t)=e^{y X}
$$

with smooth convergence on compact subsets of $S^{1} \times[0, \infty)$.

Proof. The proof is similar to that in Lot10, Propositions 4.39 and 4.79]; see also [FIN05, Theorem 1.3].

We first construct a positive solution $\widetilde{u}_{\infty}$ of the conjugate heat equation

$$
\frac{\partial u}{\partial t}=-\Delta u-\frac{1}{4} g^{y y} \operatorname{Tr}\left(\left(G^{-1} G_{, y}\right)^{2}\right) u
$$

that is defined for $t \in[0, \infty)$. To do so, note that if $u$ is a solution to (3.36) then $\int_{S^{1}} u \sqrt{g_{y y}} d y$ is constant in $t$. Let $\left\{t_{j}\right\}_{j=1}^{\infty}$ be a sequence of times going to infinity. Let $\widetilde{u}_{j}(\cdot)$ be a solution to (3.36) on the interval $\left[0, t_{j}\right]$ with initial condition $\widetilde{u}_{j}\left(t_{j}\right)=\frac{1}{L\left(t_{j}\right)}$. One shows that one can extract a subsequence of the $\widetilde{u}_{j}$ 's that converges smoothly on compact subsets of $S^{1} \times[0, \infty)$ to a positive solution $\widetilde{u}_{\infty}(\cdot)$ of (3.36). 
Define $\widetilde{f}_{\infty}(\cdot)$ by $\widetilde{u}_{\infty}(t)=(4 \pi t)^{-\frac{n}{2}} e^{-\widetilde{f}_{\infty}(t)}$. Put

$$
\begin{aligned}
& W_{+}(G, g, \tilde{f}, t)= \\
& \int_{S^{1}}\left[t\left(|\nabla \widetilde{f}|^{2}-\frac{1}{4} g^{y y} \operatorname{Tr}\left(\left(G^{-1} G_{, y}\right)^{2}\right)\right)-\widetilde{f}+1\right](4 \pi t)^{-\frac{1}{2}} e^{-\widetilde{f}} \sqrt{g_{y y}} d y .
\end{aligned}
$$

From [Lot10, Proposition 4.64],

$$
\begin{aligned}
& \frac{d}{d t} W_{+}\left(G(t), g(t), \widetilde{f}_{\infty}(t), t\right)= \\
& \frac{t}{2} \int_{S^{1}} \operatorname{Tr}\left(\left(G^{-1}\left(\triangle G-g^{y y} G_{, y} G^{-1} G_{, y}-g^{y y} G_{, y} \widetilde{f}_{\infty, y}\right)\right)^{2}\right) \widetilde{u}_{\infty} \sqrt{g_{y y}} d y+ \\
& 2 t \int_{S^{1}} g^{y y} g^{y y}\left(-\frac{1}{4} \operatorname{Tr}\left(\left(G^{-1} G_{, y}\right)^{2}\right)+\tilde{f}_{; y y}+\frac{1}{2 t} g_{y y}\right)^{2} \widetilde{u}_{\infty} \sqrt{g_{y y}} d y .
\end{aligned}
$$

In particular, $W_{+}\left(G(t), g(t), \widetilde{f}_{\infty}(t), t\right)$ is monotonically nondecreasing in $t$. Put

$$
W_{\infty}=\lim _{t \rightarrow \infty} W_{+}\left(G(t), g(t), \widetilde{f}_{\infty}(t), t\right)
$$

which at the moment could be infinity.

Using the curvature bound from Proposition 3.6 and the diameter bounds from Lemmas 3.8 and 3.12, one shows that after passing to a subsequence, $\lim _{j \rightarrow \infty}\left(g_{s_{j}}(\cdot), G_{s_{j}}(\cdot)\right)$ exists in the topology of smooth convergence on compact subsets of $S^{1} \times[0, \infty)$, and equals a solution $\left(g_{\infty}(\cdot), G_{\infty}(\cdot)\right)$ of (3.8) on a circle of time-1 length

$$
L_{\infty}=\lim _{t \rightarrow \infty} \frac{L(t)}{\sqrt{t}}
$$

(The notion of convergence allows for $j$-dependent diffeomorphisms of $S^{1}$.)

Put $u_{j}(t)=\widetilde{u}_{\infty}\left(t+s_{j}\right)$. After passing to a subsequence, we can assume that $\lim _{j \rightarrow \infty} u_{j}(t)=u_{\infty}(t)$ for some solution $u_{\infty}(\cdot)$ to (3.36) (relative to $g_{\infty}(\cdot)$ and $\left.G_{\infty}(\cdot)\right)$, with smooth convergence on compact subsets of $S^{1} \times[0, \infty)$. Define $f_{\infty}(\cdot)$ by $u_{\infty}(t)=(4 \pi t)^{-\frac{n}{2}} e^{-f_{\infty}(t)}$. Then for all $t$, we have $W_{+}\left(G_{\infty}(t), g_{\infty}(t), f_{\infty}(t), t\right)=W_{\infty}$. In particular, $W_{\infty}<\infty$. 
From (3.38), we obtain

$$
\begin{aligned}
\triangle G_{\infty}-g_{\infty}^{y y} G_{\infty, y} G_{\infty}^{-1} G_{\infty, y}-g_{\infty}^{y y} G_{\infty, y} f_{\infty, y} & =0 \\
-\frac{1}{4} \operatorname{Tr}\left(\left(G_{\infty}^{-1} G_{\infty, y}\right)^{2}\right)+f_{\infty ; y y}+\frac{1}{2 t} g_{\infty, y y} & =0 .
\end{aligned}
$$

From [Lot10, Proposition 4.80], there is a traceless symmetric matrix $X$ so that $g_{\infty, y y}(y, t)=\frac{t}{2} \operatorname{Tr}\left(X^{2}\right)$ and $G_{\infty}(y, t)=e^{y X}$. Here $y$ is a parametrization of $S^{1}$ whose time-1 velocity is $L_{\infty}$. For each $j$, we had $G_{s_{j}}(y+1, t)=H^{T} G_{s_{j}}(y, t) H$. Hence $G_{\infty}(y+1, t)=H^{T} G_{\infty}(y, t) H$ and so $e^{X}=H^{T} H$.

We now prove part (ii) of Theorem 1.6. From Proposition 3.13, for any $K \in \mathbb{Z}^{+}$and any $\epsilon>0$, there is some $t_{0}<\infty$ such that $\left(\frac{g_{y y}\left(y, t_{0}\right)}{t_{0}}, G\left(y, t_{0}\right)\right)$ is $\epsilon$-close in the $C^{K}$-norm to $\left(\frac{1}{2} \operatorname{Tr}\left(X^{2}\right), e^{y X}\right)$. From the local stability result of [Kno09, Theorem 3], after an overall reparametrization of $S^{1}$, we have

$$
\begin{aligned}
\lim _{t \rightarrow \infty} \frac{g_{y y}(y, t)}{t} & =\frac{1}{2} \operatorname{Tr}\left(X^{2}\right), \\
\lim _{t \rightarrow \infty} G(y, t) & =e^{y X} .
\end{aligned}
$$

The convergence is exponentially fast in the variable $\ln (t)$, i.e. powerlaw fast in $t$. (Strictly speaking, the result in [Kno09, Theorem 3] is for the modified Ricci flow (3.8) but there is a similar result for the unmodified Ricci flow (3.7).)

This proves Theorem 1.6.

Remark 3.14. Suppose that $H$ is parabolic. By Corollary 3.2 , the fiber volumes are uniformly bounded above and below by positive constants. After pulling back to a double cover of the base $S^{1}$, if necessary, we can assume that $\operatorname{Tr}(H)=2$. If $v \in \mathbb{R}^{2}$ is a nonzero $H$-invariant vector then (3.31) gives a uniform upper bound on the squared length $\langle v, G(y, t) v\rangle$.

Lemma 3.7 implies that the length $L(t)$ of the base circle is monotonically nondecreasing in $t$. We claim that $\lim _{t \rightarrow \infty} \frac{L(t)}{\sqrt{t}}=0$. If not then we would conclude from the proof of Proposition 3.13 that $N$ has a Sol-structure, which contradicts the topological fact that it has a Nil-structure.

If we knew that the diameters of the $T^{2}$-fibers were $O(\sqrt{t})$ then we could conclude from Lemma 2.13 and [Lot10, Theorem 1.2.2] that the pullback Ricci flow solution on the universal cover $\widetilde{N}$ approaches the Nil expanding soliton. Based on the calculation in the locally homogeneous 
Nil case, as in [Lot07, Subsubsection 3.3.3], we expect that both $L(t)$ and the fiber diameters are $O\left(t^{\frac{1}{6}}\right)$.

\section{REFERENCES}

[Cao05] Xiaodong Cao. Isoperimetric estimate for the Ricci flow on $S^{2} \times S^{1}$. Comm. Anal. Geom., 13(4):727-739, 2005.

[CFG92] Jeff Cheeger, Kenji Fukaya, and Mikhael Gromov. Nilpotent structures and invariant metrics on collapsed manifolds. J. Amer. Math. Soc., $5(2): 327-372,1992$.

[CG90] Jeff Cheeger and Mikhael Gromov. Collapsing Riemannian manifolds while keeping their curvature bounded. II. J. Differential Geom., 32(1):269-298, 1990.

[CIJ90] Mauro Carfora, James Isenberg, and Martin Jackson. Convergence of the Ricci flow for metrics with indefinite Ricci curvature. J. Differential Geom., 31(1):249-263, 1990.

[CK04] Bennett Chow and Dan Knopf. The Ricci flow: an introduction, volume 110 of Mathematical Surveys and Monographs. American Mathematical Society, Providence, RI, 2004.

[CLN06] Bennett Chow, Peng Lu, and Lei Ni. Hamilton's Ricci flow, volume 77 of Graduate Studies in Mathematics. American Mathematical Society, Providence, RI, 2006.

[CT06] Jeff Cheeger and Gang Tian. Curvature and injectivity radius estimates for Einstein 4-manifolds. J. Amer. Math. Soc., 19(2):487-525 (electronic), 2006.

[FIN05] Michael Feldman, Tom Ilmanen, and Lei Ni. Entropy and reduced distance for Ricci expanders. J. Geom. Anal., 15(1):49-62, 2005.

[Fuk89] Kenji Fukaya. Collapsing Riemannian manifolds to ones with lower dimension. II. J. Math. Soc. Japan, 41(2):333-356, 1989.

[GIK02] Christine Guenther, James Isenberg, and Dan Knopf. Stability of the Ricci flow at Ricci-flat metrics. Comm. Anal. Geom., 10(4):741-777, 2002.

[Gro78] M. Gromov. Almost flat manifolds. J. Differential Geom., 13(2):231-241, 1978.

[Ham95] Richard S. Hamilton. The formation of singularities in the Ricci flow. In Surveys in differential geometry, Vol. II (Cambridge, MA, 1993), pages 7-136. Int. Press, Cambridge, MA, 1995.

[HI93] Richard Hamilton and James Isenberg. Quasi-convergence of Ricci flow for a class of metrics. Comm. Anal. Geom., 1(3-4):543-559, 1993.

[IJ92] James Isenberg and Martin Jackson. Ricci flow of locally homogeneous geometries on closed manifolds. J. Differential Geom., 35(3):723-741, 1992.

[KL08] Bruce Kleiner and John Lott. Notes on Perelman's papers. Geom. Topol., 12(5):2587-2855, 2008.

[KM01] Dan Knopf and Kevin McLeod. Quasi-convergence of model geometries under the Ricci flow. Comm. Anal. Geom., 9(4):879-919, 2001.

[Kno00] Dan Knopf. Quasi-convergence of the Ricci flow. Comm. Anal. Geom., $8(2): 375-391,2000$.

[Kno09] Dan Knopf. Convergence and stability of locally $\mathbb{R}^{N}$-invariant solutions of Ricci flow. J. Geom. Anal., 19(4):817-846, 2009. 
RICCI FLOW ON THREE-DIMENSIONAL MANIFOLDS WITH SYMMETRY 33

[Lis08] Bernhard List. Evolution of an extended Ricci flow system. Comm. Anal. Geom., 16(5):1007-1048, 2008.

[Lot07] John Lott. On the long-time behavior of type-III Ricci flow solutions. Math. Ann., 339(3):627-666, 2007.

[Lot10] John Lott. Dimensional reduction and the long-time behavior of Ricci flow. Comment. Math. Helv., 85(3):485-534, 2010.

[Per02] Grisha Perelman. The entropy formula for the Ricci flow and its geometric applications. http://arxiv.org/abs/math.DG/0211159/, 2002.

[Ron07] Xiaochun Rong. Collapsed manifolds with bounded sectional curvature and applications. In Surveys in differential geometry. Vol. XI, volume 11 of Surv. Differ. Geom., pages 1-23. Int. Press, Somerville, MA, 2007.

[Sco83] Peter Scott. The geometries of 3-manifolds. Bull. London Math. Soc., 15(5):401-487, 1983.

Department of Mathematics, University of California - Berkeley, BERKELEY, CA 94720-3840, USA

E-mail address: lott@math.berkeley.edu

Department of Mathematics, Rutgers University, Piscataway, NJ 08854-8019, USA

E-mail address: natasas@math.rutgers.edu 